\title{
Large deviations and the maximum entropy principle for marked point random fields *
}

\author{
Hans-Otto Georgii ${ }^{1}$ and Hans Zessin ${ }^{2}$ \\ ${ }^{1}$ Mathematisches Institut, Ludwig-Maximilians-Universität München, Theresienstrasse 39, \\ W-8000 München 2, Germany \\ ${ }^{2}$ Fakultät für Mathematik, Universität Bielefeld, Universitätsstrasse, W-4800 Bielefeld 1, \\ Germany
}

Received March 5, 1992; in revised form December 23, 1992

Summary. We establish large deviation principles for the stationary and the individual empirical fields of Poisson, and certain interacting, random fields of marked point particles in $\mathbb{R}^{d}$. The underlying topologies are induced by a class of not necessarily bounded local functions, and thus finer than the usual weak topologies. Our methods yield further that the limiting behaviour of conditional Poisson distributions, as well as certain distributions of Gibbsian type, is governed by the maximum entropy principle. We also discuss various applications and examples.

\section{Introduction}

The theory of large deviations provides appropriate probabilistic tools for some fundamental problems of Equilibrium Statistical Mechanics: the existence of pressure in the thermodynamic limit, its relation to entropy, the typical behaviour of extensive quantities under energy constraints, and the equivalence of Gibbs ensembles. In fact, many of the basic ideas of this theory were developed first in Statistical Physics, and the progress in the last two decades was mainly to reveal the universality of these ideas in quite a number of probabilistic areas, and to find the proper general perspectives and techniques. Although it is only natural to apply these achievements to the original problems of Statistical Mechanics, this seems to be done so far only in the context of lattice systems (see $[3,8,17]$ and the literature cited there) and not for systems of particles in Euclidean space. It is true that such continuous systems can be viewed as lattice systems, but the class of interactions which can be handled in this way is rather restricted, see [17]. It is the object of this paper to initiate the study of large deviation principles for continuous systems by means of their own natural properties, in particular by the powerful theory of Palm measures. (After

* Supported by the Deutsche Forschungsgemeinschaft 
completion of this work we learned that weaker results in the same direction are obtained independently in a part of [14].)

The basic objects which we consider are the following. Let $\omega$ be a configuration of particles in $\mathbb{R}^{d}$, i.e., in the case of unmarked particles a locally finite subset of $\mathbb{R}^{d}$. (Later on, the particles will be allowed to have a mark describing their type or internal degrees of freedom.) Also, let $\left(\Lambda_{n}\right)$ be a fixed sequence of cubes increasing to $\mathbb{R}^{d}$. We will be concerned with the asymptotic behaviour of two different types of empirical fields of a configuration $\omega$ in $A_{n}$ as $n \rightarrow \infty$. On the one hand, we consider the familiar stationary empirical fields

$$
R_{n, \omega}=\left|\Lambda_{n}\right|^{-1} \int_{A_{n}} \delta_{3_{x} \omega^{(n)}} d x .
$$

Here we write $\left|A_{n}\right|$ for the volume of $A_{n}, \vartheta_{x}: \omega \rightarrow \omega-x$ denotes the spatial translation of configurations by the vector $-x$, and we use the standard trick of replacing $\omega$ by the periodic continuation $\omega^{(n)}$ of its restriction to $A_{n}$. This periodization has the advantage of making $R_{n, \omega}$ stationary (i.e. invariant under translations), and makes no difference in the limit $n \rightarrow \infty$. Besides the simple spatial average appearing in (1.1) one can also form an average over all particle positions in $A_{n}$. This idea leads to the measure

$$
R_{n, \omega}^{0}=\left|A_{n}\right|^{-1} \sum_{x \in \omega \cap A_{n}} \delta_{\vartheta_{x} \omega^{(n)}}
$$

which will be called the individual empirical field in $\Lambda_{n}$. Particle-position averages as in (1.2) are of primary interest in Statistical Physics because the energy per volume relative to any particle interaction can be written as such an average. They also play a major role in the statistical analysis of point processes, $\mathrm{cf}$. [10]. As a matter of fact, $R_{n, \omega}^{0}$ is intimately related to $R_{n, \omega}$. Namely, $R_{n, \omega}^{0}$ is just the Palm measure of the stationary point random field $R_{n, \omega}$. This key observation will allow us to obtain all results on the asymptotics of $R_{n, \omega}^{0}$ as simple corollaries to the corresponding results for $R_{n, \omega}$.

Here is an outline of our main results. Let $Q \circ R_{n}^{-1}$ denote the distribution of the random probability measure $R_{n}: \omega \rightarrow R_{n, \omega}$ when the particle configuration $\omega$ is distributed according to the stationary Poisson point random field $Q$ of a given intensity. Similarly, let $Q \circ\left(R_{n}^{0}\right)^{-1}$ be the distribution of the random measure $R_{n}^{0}: \omega \rightarrow R_{n, \omega}^{0}$ under $Q$. Our basic result is a large deviation principle for the distributions $Q \circ R_{n}^{-1}$ in the limit $n \rightarrow \infty$. The rate function $I$ is simply the (negative) specific entropy relative to $Q$, and the underlying topology is chosen in such a way that the mapping from the stationary point random fields to their Palm measures becomes continuous, even if the latter are equipped with the weak* topology associated with the class of all bounded local functions. The large deviation principle for the sequence $Q \circ\left(R_{n}^{0}\right)^{-1}$ can therefore be derived from the contraction principle. Also, a general extension principle of large deviation theory allows us to replace the Poisson point random field $Q$ by a point random field of Gibbsian type at least if the interaction satisfies some (unfortunately rather restrictive) stability condition.

The basic result for $Q \circ R_{n}^{-1}$ is proven along the general lines of [8]. This method shows the intimate connection between the principles of large deviations and maximum entropy. That is, with little additional effort we also obtain a result on the limiting behaviour of conditional probabilities of $Q$ under condi- 
tions on $R_{n}$ or $R_{n}^{0}$, and of Gibbsian probabilities with periodic boundary condition. The limits are precisely those which maximize the specific entropy under the given constraint, or which minimize the specific free energy, thus giving rise to what is known in Statistical Mechanics as the equivalence of ensembles.

The precise statement of the results above is given in Sect. 3. This section also contains two applications, namely the principles of large deviations and maximum entropy for the empirical distribution $L_{n}$ of particle marks and, in the one-dimensional case, for the empirical process $R_{n}^{\#}$ of particle spacings. Section 4 contains a number of illustrating examples, including a gas model of Curie-Weiss type, the Widom-Rowlinson model, and one-dimensional models with nearest-particle interaction (which are related to renewal processes). Most proofs are deferred to Sect. 5. In Sect. 2 below we introduce the set-up, recall a number of basic facts, establish the Palm relation between the empirical fields $R_{n}$ and $R_{n}^{0}$, and discuss their ergodic behaviour.

\section{Preliminaries}

\subsection{Marked point random fields}

To describe random systems of marked points (or particles) in the space $\mathbb{R}^{d}$ we proceed as follows. Let $S=\mathbb{R}^{d}, \mathscr{B}_{S}$ be the Borel $\sigma$-algebra on $S$, and $\lambda$ the Lebesgue measure on $\left(S, \mathscr{B}_{S}\right)$. We often write $|\Delta|$ for $\lambda(\Delta)$. By $\mathscr{C}$ we denote the set of all open cubes in $S$ of the form $A=\prod_{i=1}^{d}\left(q_{i}, q_{i}+p\right)$ with $q=\left(q_{1}, \ldots, q_{d}\right) \in S$ and $p>0 . p$ is called the side length of $A$. We also let $\left(A_{n}\right)$ be a fixed sequence of cubes $A_{n} \in \mathscr{C}$ with side lengths $p_{n}$ such that $\Lambda_{n} \uparrow \mathbb{R}^{d}$ as $n \rightarrow \infty$.

Furthermore we introduce a mark space $E$ which is equipped with a complete, separable metric $d_{E}$, the associated Borel $\sigma$-algebra $\mathscr{B}_{E}$, and a finite a priori measure $\mu$ on $E$ with $\mu(E)>0$. The phase space for a particle is $X=S \times E$. A standard choice for a product metric $d_{X}$ turns $X$ into a complete separable metric space with Borel $\sigma$-algebra $\mathscr{B}_{X}=\mathscr{B}_{S} \otimes \mathscr{B}_{E}$.

Examples. (1) If $E$ is a singleton, $X$ can be identified with $S$. This is the case when the particles have no mark. The total mass $\mu(E)>0$ of $\mu$ then just plays the role of an intensity (= particle density) parameter.

(2) If $E=\mathbb{R}^{d}$ and $\mu$ is a centered Gaussian distribution having a positive multiple of the identity matrix as covariance matrix, we are in the classical physical case where each particle is equipped with a velocity having a Maxwellian distribution.

(3) If $E=\mathscr{C}\left([0, \infty), \mathbb{R}^{d}\right)$ and $\mu$ is the Wiener measure, then each particle is equipped with a realization of Brownian motion which may be thought of as a description of its time evolution.

As should be evident from Examples (2) and (3), it is often reasonable to consider not only bounded functions on $E$ but also unbounded functions satisfying a suitable growth condition. To this end we fix a measurable function $\psi: E \rightarrow[1, \infty)$ which is related to $\mu$ via the condition

$$
\mu\left(e^{a \psi}\right)=\int e^{a \psi} d \mu<\infty \quad \text { for all } a>0
$$


and we shall confine our attention to functions on $E$ which are dominated by a multiple of $\psi$. Clearly, a possible choice is $\psi \equiv 1$.

A configuration of marked particles in $\mathbb{R}^{d}$ without multiple occupancies can be described by a pair $\left(\xi,\left(u_{x}\right)_{x \in \xi}\right)$, where the set $\xi \subset S$ of occupied places is locally finite, in that $\xi \cap A$ is finite for each $A \in \mathscr{C}$, and $u_{x} \in E$ is the mark of the particle at position $x \in \xi$. It is convenient to describe such a configuration by the counting measure

$$
\omega=\sum_{x \in \xi} \delta_{\left(x, u_{x}\right)}
$$

on $\left(X, \mathscr{B}_{X}\right)$. Counting measures of this form (i.e., without multiple occupancies) are called simple. Thus our configuration space is the set $\Omega$ of all simple counting measures on $\left(X, \mathscr{B}_{X}\right)$. (This choice of $\Omega$ is natural because the Poisson point random fields considered later are supported on configurations without multiple occupancies.) For each $B \in \mathscr{B}_{X}$, the counting variable $N(B): \omega \rightarrow \omega(B)$ on $\Omega$ describes the number of particles which are such that the pair (position, mark) belongs to $B$. In particular, for each $A \in \mathscr{B}_{S}$ we write $N_{\Lambda}=N(A \times E)$ for the number of particles which are located in $A$. Also, for each $A \in \mathscr{B}_{S}$ and measurable $h: E \rightarrow[0, \infty)$ we introduce the variable

$$
N_{A}^{h}=N_{A}(h): \omega \rightarrow \int_{A \times E} h(u) \omega(d x, d u) .
$$

A particularly important role will be played by the random variables $N_{\Lambda}^{\psi}, A \in \mathscr{C}$. Clearly, if $\psi=1$ then $N_{A}^{\psi}=N_{A}$. The space $\Omega$ will be equipped with the $\sigma$-algebra $\mathscr{F}=\sigma\left(N(B): B \in \mathscr{B}_{X}\right)$ generated by all counting variables. It is well-known [11] that $\mathscr{F}$ is the Borel $\sigma$-algebra for the Polish topology $\tau_{\Omega}$ which is generated by the variables $\omega \rightarrow \int g d \omega$ where $g: X \rightarrow \mathbb{R}$ is bounded and continuous with spatially bounded support.

For each $A \in \mathscr{C}$ we shall consider also the set of configurations of particles which are located in $A$, namely $\Omega_{A}=\left\{N_{\Lambda^{c}}=0\right\} . \Omega_{A}$ is a $G_{\delta}$ and thus again Polish. The associated Borel $\sigma$-field $\mathscr{F}_{A}$ on $\Omega_{A}$ coincides with the restriction of $\mathscr{F}$ to $\Omega_{A}$, and the restriction mapping $r_{\Lambda}: \Omega \rightarrow \Omega_{A}, r_{A}(\omega)=\omega(\cdot \cap A \times E)$, is $\mathscr{F}-$ $\mathscr{F}_{\Lambda}$-measurable. Furthermore, $\mathscr{F}=\sigma\left(\bigcup_{n \geqq 1} r_{A_{n}}^{-1} \mathscr{F}_{A_{n}}\right)$.

We also need to introduce the shift group $\Theta=\left(\vartheta_{x}\right)_{x \in S}$ acting on $\Omega$ via $\left(\vartheta_{x} \omega\right)(B)=\omega(B+x)$, where $B+x=\{(y+x, u):(y, u) \in B\}$. The mapping $\vartheta: S \times \Omega$ $\rightarrow \Omega, \vartheta(x, \omega)=\vartheta_{x} \omega$, and all $\vartheta_{x}$ are measurable [11].

Any probability measure $P$ on $(\Omega, \mathscr{F})$ is called a (simple) marked point random field on $X$. We often write $P(f)$ for the $P$-integral of a measurable function $f$ on $\Omega . P$ is said to be stationary if $P \circ \vartheta_{x}^{-1}=P$ for each $x \in S$. We say $P$ is of first $\psi$-order if $P\left(N_{\Lambda}^{\psi}\right)<+\infty$ for each $\Lambda \in \mathscr{C}$. Since for a stationary $P$ the measure $\mathscr{B}_{S} \ni \Delta \rightarrow P\left(N_{d}^{W}\right)$ is invariant under translations, a stationary $P$ is of first $\psi$-order if and only if there exists a number $z^{\psi}(P)<\infty$, the $\psi$-intensity of $P$, such that $P\left(N_{\Delta}^{\psi}\right)=z^{\psi}(P)|\Delta|$ for all $\Delta \in \mathscr{B}_{S}$. Since $\psi \geqq 1$, it then also follows that $P\left(N_{A}\right)=z(P)|\Delta|$ for all $\Delta \in \mathscr{B}_{S}$ and a finite number $z(P)$ which is called the intensity of $P$. We denote by $\mathscr{P}$ the set of all marked point random fields on $X$ of first $\psi$-order, and we write $\mathscr{P}_{\mathbb{P}}$ for the set of all stationary point random fields on $X$ with finite $\psi$-intensity. The natural $\sigma$-fields on $\mathscr{P}$ and $\mathscr{P}_{\theta}$, which we denote by $\Sigma$ and $\Sigma_{\theta}$, are defined as the smallest $\sigma$-fields for which all evalua- 
tion mappings $e_{A}: P \rightarrow P(A), A \in \mathscr{F}$, are measurable. It is obvious that for each measurable function $f$ on $\Omega$ the mapping $e_{f}: P \rightarrow P(f)$ is also measurable, provided it is well-defined. $\Sigma$ is the Borel $\sigma$-field for the weak topology $\tau_{w}$ on $\mathscr{P}$ based on the topology $\tau_{\Omega}$, and the same holds for $\Sigma_{\Theta}$ since $\mathscr{P}_{\Theta}$ is $\tau_{w}$-closed, cf. [11].

Nevertheless, in this paper we shall consider a finer topology $\tau_{\mathscr{L}}$ on $\mathscr{P}$ defined as follows. A function $f: \Omega \rightarrow \mathbb{R}$ is called local if $f$ is measurable relative to $r_{A}^{-1} \mathscr{F}_{\Lambda}$ for some $A \in \mathscr{C} . f$ will be called tame if $|f| \leqq b\left(1+N_{\Lambda}^{\psi}\right)$ for some $\Lambda \in \mathscr{C}$ and a constant $b<\infty$. We let $\mathscr{L}$ denote the linear space of all tame local functions. The topology $\tau_{\mathscr{L}}$ of local convergence is then defined as the smallest topology on $\mathscr{P}$ for which the mappings $e_{f}: P \rightarrow P(f), f \in \mathscr{L}$, are continuous. It follows easily from the portmanteau theorem that indeed $\tau_{\mathscr{L}} \supset \tau_{w}$.

From now on we assume that $\mathscr{P}$ is equipped with $\tau_{\mathscr{L}}$. The $\tau_{\mathscr{L}}$-closed subset $\mathscr{P}_{\Theta}$ will be equipped with the induced topology. Observe that the $\psi$-intensity functional $z^{\psi}$ and the intensity functional $z$ on $\mathscr{P}_{\boldsymbol{P}}$ are $\tau_{\mathscr{P}}$-continuous.

Next we recall the concept of Palm measure of a stationary marked point random field. We summarize its basic properties in a remark which can be proved in complete analogy to the well-known unmarked case [12].

Remark 2.1 For each $P \in \mathscr{P}_{\Theta}$ there exists a unique finite measure $P^{0}$ on $\left(\Omega^{0}, \mathscr{F}^{0}\right)$ $=\left(E \times \Omega, \mathscr{B}_{E} \otimes \mathscr{F}\right)$, the Palm measure of $P$, such that

$$
\iint f\left(x, u, \vartheta_{x} \omega\right) \omega(d x, d u) P(d \omega)=\iint f(x, u, \omega) d x P^{0}(d u, d \omega)
$$

for all measurable functions $f: S \times E \times \Omega \rightarrow[0, \infty)$, and therefore

$$
P^{0}(g)=\iint 1_{C}(x) g\left(u, \vartheta_{x} \omega\right) \omega(d x, d u) P(d \omega)
$$

for all measurable $g: E \times \Omega \rightarrow[0, \infty)$ and arbitrary $C \in \mathscr{B}_{S}$ with $|C|=1$. In particular, $P^{0}$ is supported on the set $\{(u, \omega) \in E \times \Omega: \omega\{(0, u)\}=1\}$, and for the marginal $\mu_{P}=P^{0}(\cdot \times \Omega)$ of $P^{0}$ on $\left(E, \mathscr{B}_{E}\right)$ we have

$$
P(N(B))=\lambda \otimes \mu_{P}(B) \quad \text { for all } B \in \mathscr{B}_{X}
$$

and $P^{0}(E \times \Omega)=\mu_{P}(E)=z(P), P^{0}(\psi \otimes 1)=\mu_{P}(\psi)=z^{\psi}(P) . \mu_{P}$ is called the mark intensity measure of $P$.

We now consider the mapping $\pi^{0}: P \rightarrow P^{0}$ on $\mathscr{P}_{\theta}$. By definition, $P^{0}$ belongs to the set $\mathscr{M}^{0}$ of all finite measures on $\left(\Omega^{0}, \mathscr{F}^{0}\right)$ for which the function $\psi \otimes 1$ : $(u, \omega) \rightarrow \psi(u)$ on $\Omega^{0}$ is integrable. We call a function $g: \Omega^{0} \rightarrow \mathbb{R}$ local if it is measurable with respect to $\mathscr{B}_{E} \otimes r_{A}^{-1} \mathscr{F}_{A}$ for some $\Lambda \in \mathscr{C}$, and we write $\mathscr{L}^{0}$ for the set of all local functions $g$ on $\Omega^{0}$ which are such that $|g| \leqq b \psi \otimes 1$ for some

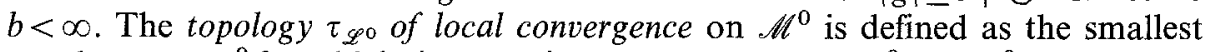
topology on $\mathscr{M}^{0}$ for which the mappings $e_{g}: m \rightarrow m(g), g \in \mathscr{L}^{0}$, on $\mathscr{M}^{0}$ are continuous. We also equip $\mathscr{M}^{0}$ with the evaluation $\sigma$-algebra $\Sigma^{0}$ generated by the mappings $e_{A}, A \in \mathscr{F}^{0}$.

Remark 2.2 The mapping $\pi^{0}: \mathscr{P}_{\otimes} \rightarrow \mathscr{M}^{0}$ defined by $\pi^{0}(P)=P^{0}$ is affine, injective, measurable and $\tau_{\mathscr{L}}-\tau_{\mathscr{L} 0}$-continuous. 
Proof. It follows readily from (2.4) that $\pi^{0}$ is affine and measurable. Also, for any $g \in \mathscr{L}^{0}$ and bounded $C$ the inner integral on the right side of (2.4) belongs to $\mathscr{L}$. This shows that $\pi^{0}$ is continuous. Finally, (2.3) implies that $P$ can be recovered from $P^{0}$, cf. Lemma 12.1.III of [2].

\subsection{The empirical fields}

For each $n$ and $\omega \in \Omega_{A_{n}}$ we denote by $\omega^{(n)}$ the periodic continuation of $\omega$; i.e., $\omega^{(n)}=\sum_{i \in p_{n} \mathbb{Z}^{d}} \vartheta_{i} \omega$. Observe that the mapping $\omega \rightarrow \omega^{(n)}$ is $\mathscr{F}_{\Lambda_{n}}-\mathscr{F}$-measurable. The associated stationary empirical field $R_{n, \omega}$ is defined by Eq. (1.1). The definition of the individual empirical field $R_{n, \omega}^{0}$, however, becomes more subtle in the presence of a mark space $E$. $R_{n, \omega}^{0}$ is now defined as a measure on $\Omega^{0}$ rather than on $\Omega$. Namely, in the notation of (2.2),

$$
R_{n, \omega}^{0}=\left|\Lambda_{n}\right|^{-1} \int_{A_{n} \times E} \delta_{\left(u, \vartheta_{x} \omega^{(n)}\right)} \omega(d x, d u)=\left|\Lambda_{n}\right|^{-1} \sum_{x \in \xi} \delta_{\left(u_{x}, Q_{x} \omega^{(n)}\right)} .
$$

Remark 2.3 For all $n$ and $\omega$ we have

(1) $R_{n, \omega} \in \mathscr{P}_{\theta}, R_{n, \omega}^{0} \in \mathscr{M}^{0}, z^{\psi}\left(R_{n, \omega}\right)=\left|A_{n}\right|^{-1} N_{\Lambda_{n}}^{\psi}(\omega)$;

(2) $R_{n}: \omega \rightarrow R_{n, \omega}$ is $\mathscr{F}_{A_{n}}-\Sigma_{\Theta}$-measurable, and

$R_{n}^{0}: \omega \rightarrow R_{n, \omega}^{0}$ is $\mathscr{F}_{A_{n}}-\Sigma^{0}$-measurable;

(3) $R_{n, \omega}^{0}$ is the Palm measure of $R_{n, \omega}$.

Proof. (1) The stationarity of $R_{n, \omega}$ follows from the periodicity of $\omega^{(n)}$. By Remark 2.1 and assertion (3), $\quad z^{\psi}\left(R_{n, \omega}\right)=R_{n, \omega}^{0}(\psi \otimes 1)=\left|A_{n}\right|^{-1} \omega\left(1_{A_{n}} \otimes \psi\right)=$ $\left|\Lambda_{n}\right|^{-1} N_{\Lambda_{n}}^{\psi}(\omega)$ which is finite because $\omega\left(A_{n} \times E\right)$ is finite.

(2) Obvious.

(3) We verify Eq. (2.3). Let $f: S \times E \times \Omega \rightarrow \mathbb{R}_{+}$be measurable. Then

$$
\begin{aligned}
& \left|A_{n}\right| \iint f\left(x, u, \vartheta_{x} \zeta\right) \zeta(d x, d u) R_{n, \omega}(d \zeta) \\
& \quad=\iint_{A_{n}} f\left(x-y, u, \vartheta_{x} \omega^{(n)}\right) \omega^{(n)}(d x, d u) d y \\
& \quad=\sum_{i \in p_{n} \mathbb{Z}^{d}} \iint f\left(x+i-y, u, \vartheta_{x+i} \omega^{(n)}\right) \omega(d x, d u) d y .
\end{aligned}
$$

This equals

$$
\iint_{s} f\left(x-y, u, \vartheta_{x} \omega^{(n)}\right) \omega(d x, d u) d y
$$

because $\vartheta_{x+i} \omega^{(n)}=\vartheta_{x} \omega^{(n)}$ for all $i \in p_{n} \mathbb{Z}^{d}$. Since Lebesgue measure is reflection invariant we arrive at the expression

$$
\begin{aligned}
& \iint f\left(y, u, \vartheta_{x} \omega^{(n)}\right) d y \omega(d x, d u) \\
& \quad=\left|A_{n}\right| \iint f(y, u, \zeta) d y R_{n, \omega}^{0}(d u, d \zeta) .
\end{aligned}
$$


The remark above shows that the periodization of $\omega$ has great technical advantages. In the topology of local convergence the effect of this periodization becomes negligible if $n \rightarrow \infty$, as we will now show. It will be convenient to consider $R_{n}$ as an $r_{A_{n}}^{-1} \mathscr{F}_{A_{n}}$-measurable function on $\Omega$.

Remark 2.4 For each $f \in \mathscr{L}$ and $P \in \mathscr{P}_{\Theta}$,

$$
\left.\left|R_{n}(f)-\right| \Lambda_{n}\right|^{-1} \int_{\Lambda_{n}} f \circ \vartheta_{x} d x \mid \rightarrow 0
$$

in $L^{1}(P)$-norm as $n \rightarrow \infty$.

Proof. Let $\Delta \in \mathscr{C}$ be such that $f$ is $r_{\Delta}^{-1} \mathscr{F}_{\Delta}$-measurable and $|f| \leqq b\left(1+N_{\alpha}^{\psi}\right)$ for some $b>0$. Then $f\left(\vartheta_{x} \omega\right)=f\left(\vartheta_{x} \omega^{(n)}\right)$ for all $n$ and $x$ such that $\Delta+x \subset \Lambda_{n}$ and all $\omega \in \Omega$. Writing $\partial \Lambda_{n}=\left|\left\{x \in \Lambda_{n}:(\Delta+x) \backslash \Lambda_{n} \neq \emptyset\right\}\right|$, we thus obtain

$$
\begin{aligned}
& \left.\left|R_{n, \omega}(f)-\right| A_{n}\right|^{-1} \int_{\Lambda_{n}} f\left(\vartheta_{x} \omega\right) d x \mid \\
& \quad \leqq\left|A_{n}\right|^{-1} b \int_{\partial A_{n}}\left(2+N_{\Delta+x}^{\psi}\left(\omega^{(n)}\right)+N_{d+x}^{\psi}(\omega)\right) d x .
\end{aligned}
$$

Since $\left|A_{n}\right|^{-1}\left|\partial A_{n}\right| \rightarrow 0$ as $n \rightarrow \infty$, it is sufficient to prove that

$$
\int\left(N_{\Delta+x}^{\psi}\left(\omega^{(n)}\right)+N_{A+x}^{\psi}(\omega)\right) P(d \omega)=2 z^{\psi}(P)|\Delta|
$$

for all $P \in \mathscr{P}_{\Theta}$ and $x \in S$. But clearly $P\left(N_{\Delta+x}^{\psi}\right)=z^{\psi}(P)|A|$. On the other hand,

$$
\int N_{\Delta+x}^{\psi}\left(\omega^{(n)}\right) P(d \omega)=P\left(N_{\Delta_{x, n}}^{\psi}\right)=z^{\psi}(P)\left|\Delta_{x, n}\right|,
$$

where $\Delta_{x, n}=\left\{y \in A_{n}: y+i \in \Delta+x\right.$ for some $\left.i \in p_{n} \mathbb{Z}^{d}\right\}$. Since $\left|\Delta_{x, n}\right|=|\Delta+x|=|\Delta|$, the remark follows.

The empirical fields $R_{n}$ and $R_{n}^{0}$ exhibit the expected ergodic behaviour as $n \rightarrow \infty$. Indeed, suppose $P \in \mathscr{P}_{\theta}$ is ergodic, i.e., $P$ is extreme in $\mathscr{P}_{\theta}$. Wiener's multidimensional mean ergodic theorem then implies that

$$
\left|A_{n}\right|^{-1} \int_{A_{n}} f \circ \vartheta_{x} d x \rightarrow P(f) \quad \text { in } L^{1}(P)
$$

for all $f \in L^{1}(P)$. By the last remark this implies that $R_{n}(f) \rightarrow P(f)$ in $L^{1}(P)$ for all $f \in \mathscr{L}$. It follows that

$$
\tau_{\mathscr{P}}-\lim _{n \rightarrow \infty} R_{n}=P \quad \text { in } P \text {-probability. }
$$

In view of Remarks 2.2 and 2.3 we also obtain that

$$
\tau_{\mathscr{L}^{\circ}}-\lim _{n \rightarrow \infty} R_{n}^{0}=P^{0} \quad \text { in } P \text {-probability. }
$$

The aim of this paper is to show that the convergence in (2.8) and (2.9) is exponentially fast, at least if $P$ is a Poisson point random field or not too far away from such a field. The speed of this convergence can be described in terms of specific entropy. 


\subsection{Poisson random fields and specific entropy}

We let $Q$ denote the Poisson point random field on $X$ with intensity measure $\lambda \otimes \mu$. By definition, $Q$ is the unique (simple) marked point random field such that, for any choice of disjoint and spatially bounded sets $B_{1}, \ldots, B_{n}$ in $\mathscr{B}_{X}$, the counting variables $N\left(B_{1}\right), \ldots, N\left(B_{n}\right)$ are independent and Poisson distributed with parameters $\lambda \otimes \mu\left(B_{1}\right), \ldots, \lambda \otimes \mu\left(B_{n}\right)$. It is easily seen that $\mu_{Q}=\mu$ and thus $z(Q)=\mu(E)$, cf. (2.5). Note also that under $Q$ the marks of all particles are iid with distribution $\mu / \mu(E)$. Later on, we will also consider Poisson random fields with a mark intensity measure $v$ different from $\mu$. We then shall write $Q^{v}$ for the sake of distinction. Thus $Q=Q^{\mu}$.

Next, we introduce the specific entropy of any $P \in \mathscr{P}_{\Theta}$ relative to $Q$. For each $\Lambda \in \mathscr{C}$ we write $P_{A}=P \circ r_{A}^{-1}$ for the projection onto $\left(\Omega_{\Lambda}, \mathscr{F}_{A}\right)$. The relative entropy of two measures on the same measurable space is defined by

$$
I(\alpha ; \beta)= \begin{cases}\beta(1-f+f \log f) & \text { if } \alpha=f \beta \\ \infty & \text { else. }\end{cases}
$$

It is well known and easy to see that $I(\alpha ; \beta) \geqq 0$ with equality if and only if $\alpha=\beta$. For probability measures $\alpha$ and $\beta$ we have

$$
I(\alpha ; \beta)=\sup _{g}\left[\alpha(g)-\log \beta\left(e^{g}\right)\right]
$$

where the sup extends over all bounded measurable functions $g$ on the underlying space. A proof of (2.11) can be found in [19], for example. It follows readily from (2.10) that $I(\alpha ; \beta)$ equals $\alpha(1)$ times the relative entropy of the normalized measures $\alpha / \alpha(1)$ and $\beta / \beta(1)$, plus the relative entropy of the Poisson distributions with parameters $\alpha(1)$ resp. $\beta(1)$. The (negative) specific entropy or mean entropy of any $P \in \mathscr{P}_{\Theta}$ is now defined as

$$
I(P)=\lim _{n \rightarrow \infty}\left|\Lambda_{n}\right|^{-1} I\left(P_{\Lambda_{n}} ; Q_{\Lambda_{n}}\right)
$$

The following properties of $I$ can be proved in the same way as the analogous results for lattice systems, cf. [7].

Remark 2.5 (1) For all $P \in \mathscr{P}_{\Theta}$,

$$
I(P)=\sup _{\Lambda \in \mathscr{C}}|\Lambda|^{-1} I\left(P_{\Lambda} ; Q_{\Lambda}\right) .
$$

In particular, $I(P)$ does not depend on the choice of the sequence $\left(A_{n}\right)$.

(2) The functional $I: \mathscr{P}_{\oplus} \rightarrow[0, \infty]$ is affine.

A further fundamental property of $I$ is stated in the next proposition which will be proved in Sect. 5.1.

Proposition 2.6 The level sets $\{I \leqq c\}$ are compact and sequentially compact in $\tau_{\mathscr{L}}$. Thus $I$ is lower semicontinuous relative to $\tau_{\mathscr{L}}$ and any coarser topology on $\mathscr{P}$. 


\section{Statement of results}

\subsection{Large deviations}

Our main result is a large deviation principle for $R_{n}$ relative to $Q$. Large deviation principles for $R_{n}^{0}$ and certain Gibbsian point random fields then follow as an immediate consequence. In fact, we state these principles in the more general form of Laplace approximation, i.e., we consider exponential integrals of functionals $F\left(R_{n}\right)$ of $R_{n}$, or of functions which are asymptotically close to such functionals. To simplify the statement we introduce the following concept. We say that $\left\{\left(F_{n}\right), F\right\}$ is an asymptotic empirical functional, if $F: \mathscr{P}_{\Theta} \rightarrow(-\infty, \infty]$ is a function satisfying $F \geqq-b\left(1+z^{\psi}\right)$ for some finite constant $b$, and $\left(F_{n}\right)$ is a sequence of $\mathscr{F}_{A_{n}}$-measurable functions $F_{n}: \Omega_{\Lambda_{n}} \rightarrow(-\infty, \infty]$ such that

$$
\left.|| \Lambda_{n}\right|^{-1} F_{n}-F\left(R_{n}\right) \mid \leqq \delta_{n}^{F}\left(1+z^{\psi}\left(R_{n}\right)\right)
$$

for a sequence $\delta_{n}^{F} \rightarrow 0$. Here we use the convention $\infty-\infty=0$. For each $F: \mathscr{P}_{P} \rightarrow(-\infty, \infty]$ we let $F^{\mathrm{usc}}$ and $F_{\mathrm{lsc}}$ denote the semicontinuous regularizations of $F$. That is, $F_{\text {Isc }}$ is the largest lower semicontinuous minorant and $F^{\text {usc }}$ the lowest upper semicontinuous majorant of $F$.

Theorem 3.1 For any asymptotic empirical functional $\left\{\left(F_{n}\right), F\right\}$,

$$
\limsup _{n \rightarrow \infty}\left|A_{n}\right|^{-1} \log Q_{A_{n}}\left(\exp \left(-F_{n}\right)\right) \leqq-\inf \left[I+F_{\text {lsc }}\right]
$$

and

$$
\liminf _{n \rightarrow \infty}\left|A_{n}\right|^{-1} \log Q_{\Lambda_{n}}\left(\exp \left(-F_{n}\right)\right) \geqq-\inf \left[I+F^{\text {usc }}\right]
$$

Theorem 3.1 will be proved in Sect. 5.3. As an application we obtain a similar result for functionals of the individual empirical fields $R_{n}^{0}$. Since $R_{n}^{0}=\pi^{0}\left(R_{n}\right)$, this simply amounts to considering asymptotic empirical functionals with an $F$ of the form $F=G \circ \pi^{0}$ for some $G: \mathscr{M}^{0} \rightarrow(-\infty, \infty]$. In other words, we only need to apply the contraction principle [19] to the continuous injection $\pi^{0}$. This gives the following result.

Corollary 3.2 Consider an asymptotic empirical functional of the form $\left\{\left(G_{n}\right), G \circ \pi^{0}\right\}$ for some $G: \mathscr{M}^{0} \rightarrow(-\infty, \infty]$. Then

$$
\limsup _{n \rightarrow \infty}\left|A_{n}\right|^{-1} \log Q_{A_{n}}\left(\exp \left(-G_{n}\right)\right) \leqq-\inf \left[I^{0}+G_{1 \mathrm{sc}}\right]
$$

and

$$
\liminf _{n \rightarrow \infty}\left|A_{n}\right|^{-1} \log Q_{A_{n}}\left(\exp \left(-G_{n}\right)\right) \geqq-\inf \left[I^{0}+G^{\text {usc }}\right],
$$

where $I^{0}: \mathscr{M}^{0} \rightarrow[0, \infty]$ is defined by

$$
I^{0}(m)= \begin{cases}I(P) & \text { if } m=P^{0} \text { for a (necessarily unique) } P \in \mathscr{P}_{\Theta} \\ +\infty & \text { else. }\end{cases}
$$

$I^{0}$ has $\tau_{\mathscr{L}^{0}}$-compact level sets. 
It is important to note that Theorem 3.1 and Corollary 3.2 can be extended immediately to the case where the Poisson random fields $Q_{\Lambda_{n}}$ are replaced by marked point random fields of Gibbsian type allowing for a dependence of particles. These are defined in terms of an asymptotic empirical functional $\left\{\left(F_{n}\right), F\right\}$ via the formula

$$
Q_{n}^{F}=Q_{\Lambda_{n}}\left(e^{-F_{n}}\right)^{-1} e^{-F_{n}} Q_{\Lambda_{n}} .
$$

For more details on this straightforward extension we refer to Corollary 1.5 of [8], and to Boltzmann's principle in [3] and [21]. Some applications will be discussed in Sect. 4.

\subsection{The maximum entropy principle}

As we have noted in the introduction, the large deviation principle is intimately related to the maximum entropy principle which refers to the asymptotic behaviour of the Gibbsian measures $Q_{n}^{F}$ as $n \rightarrow \infty$. In this context it is convenient to think of $Q_{n}^{F}$ as an element of $\mathscr{P}$ which is supported on $\Omega_{A_{n}}$, and it is worthwhile to note that $Q_{n}^{F}$ is equal to a conditional probability of the form $Q_{A_{n}}\left(\cdot \mid R_{n} \in K\right)$ with $K \in \Sigma_{\Theta}$ when $F_{n}=\infty 1_{\left\{R_{n} \notin K\right\}}$.

Theorem 3.3 Let $\left\{\left(F_{n}\right), F\right\}$ be an asymptotic empirical functional and suppose that each $F_{n}$ is a function of $R_{n}$. If, in addition,

$$
\inf \left[I+F_{1 s c}\right]=\inf \left[I+F^{\mathrm{usc}}\right]<\infty
$$

then $\left(Q_{n}^{F}\right)$ is sequentially compact, and each accumulation point $Q_{\infty}^{F}$ of $\left(Q_{n}^{F}\right)$ is the barycenter of a Borel probability measure on the compact set

$$
M^{F}=\left\{P \in \mathscr{P}_{\Theta}: I(P)+F_{\mathrm{lsc}}(P)=\inf \left[I+F_{\mathrm{lsc}}\right]\right\} .
$$

By the last sentence we mean that $Q_{\infty}^{F}=\int P w(d P)$ for a probability measure $w$ on the Borel $\sigma$-algebra on $M^{F}$. Note that such an integral representation makes sense because the evaluation mapping $e_{A}$ is continuous for $A \in \bigcup_{A \in \mathscr{C}} r_{A}^{-1} \mathscr{F}_{A}$
and thus Borel measurable for all $A \in \mathscr{F}$.

Condition (3.6) is clearly satisfied when $F$ is continuous and not identically $+\infty$ on $\{I<\infty\}$. It is also satisfied when $F$ is convex and $\left\{F^{\mathrm{usc}}<\infty\right\} \cap\{I<\infty\}$ $\neq \emptyset$; see, e.g., $[8,21]$. If $M^{F}$ is a singleton (which certainly holds when $F$ is strictly convex) then $\left(Q_{n}^{F}\right)$ converges to the unique element of $M^{F}$. As an immediate consequence of Theorem 3.3 we obtain again its counterpart for functionals of the individual empirical fields $R_{n}^{0}$.

Corollary 3.4 Consider an asymptotic empirical functional of the form $\left\{\left(G_{n}\right), G \circ \pi^{0}\right\}$, where $G: \mathscr{M}^{0} \rightarrow(-\infty, \infty]$, and suppose each $G_{n}$ is a function of $R_{n}$ or $R_{n}^{0}$. If

$$
\inf \left[I^{0}+G_{1 s c}\right]=\inf \left[I^{0}+G^{u s c}\right]<\infty,
$$

then $\left(Q_{n}^{G}\right)$ is sequentially compact, and each accumulation point of $\left(Q_{n}^{G}\right)$ is the barycenter of a Borel probability measure on the compact set

$$
M^{G}=\left\{P \in \mathscr{P}_{\Theta}: I(P)+G_{1 \mathrm{sc}}\left(P^{0}\right)=\inf \left[I^{0}+G_{1 \mathrm{sc}}\right]\right\} .
$$


The corollary above can be used to prove the asymptotic equivalence of microcanonical and grand canonical Gibbs distributions for suitable interactions. We do not go into this here because the arguments are similar to the lattice case discussed in $[3,8]$. Some specific examples will be considered in Sect. 4.

\subsection{Application to the empirical distribution of marks}

Let $\mathscr{M}_{E}$ denote the space of all finite measures $v$ on $\left(E, \mathscr{B}_{E}\right)$ with $v(\psi)<\infty$, equipped with the topology $\tau_{\mathscr{L}_{E}}$ which is generated by the space $\mathscr{L}_{E}$ of all measurable functions $h: E \rightarrow \mathbb{R}$ with $|h| \leqq b \psi$ for some $b<\infty$. For each $n \geqq 1$ and $\omega \in \Omega_{\Lambda_{n}}$ of the form (2.2) let

$$
L_{n, \omega}=\left|A_{n}\right|^{-1} \int \delta_{u} \omega(d x, d u)=\left|A_{n}\right|^{-1} \sum_{x \in \zeta} \delta_{u_{x}} \in \mathscr{M}_{E}
$$

denote the empirical mark intensity measure of $\omega$ in $A_{n} . L_{n}: \omega \rightarrow L_{n, \omega}$ is related to the other empirical fields via the identity

$$
L_{n}=\pi_{E}\left(R_{n}^{0}\right)=\pi_{E} \circ \pi^{0}\left(R_{n}\right)=\mu_{R_{n}} .
$$

Here $\pi_{E}: \mathscr{M}^{0} \rightarrow \mathscr{M}_{E}$ is the marginal projection defined by $\pi_{E}(m)=m(\cdot \times \Omega)$, and $\mu_{R_{n}}$ is as in Remark 2.1. By the definitions of $\tau_{\mathscr{L O}}$ and $\tau_{\mathscr{L}_{E}}, \pi_{E}$ is continuous.

We consider the relative entropy functional $I_{E}: \mathscr{M}_{E} \rightarrow[0, \infty]$ defined by $I_{E}(v)=I(v ; \mu), v \in \mathscr{M}_{E} . I_{E}$ is convex and strictly convex on its effective domain $\left\{I_{E}<+\infty\right\}$. The significance of $I_{E}$ becomes clear from the proposition below which will be proved in Sect. 5.2.

Proposition 3.5 If $v \in \mathscr{M}_{E}$ then for each $P \in \mathscr{P}_{B}$ with $\mu_{P}=v$ we have

$$
I(P)=I_{v}(P)+I_{E}(v),
$$

where $I_{v}$ denotes the specific entropy relative to the Poisson point random field $Q^{v}$ on $X$ with intensity measure $\lambda \otimes v$. Hence

$$
I_{E}(v)=\inf _{P \in \mathscr{P} \Theta: \mu_{P}=v} I(P),
$$

and $Q^{v}$ is the unique probability law in $\mathscr{P}_{\mathbb{B}}$ attaining the infimum. In particular, $I_{E}$ has compact level sets.

As an obvious consequence of this proposition and Corollaries 3.2 and 3.4 we obtain the following large deviation principle of Sanov-type and a limit theorem of Csiszar-type; cf. [1].

Corollary 3.6 Let $H: \mathscr{M}_{E} \rightarrow(-\infty, \infty]$ be such that $H \geqq-b e_{\psi}$ for some $b<\infty$ and, for each $n \geqq 1, H_{n}: \Omega_{\Lambda_{n}} \rightarrow(-\infty, \infty]$ a measurable function such that

$$
\left\|\left(\left|A_{n}\right|^{-1} H_{n}-H\left(L_{n}\right)\right) /\left(1+L_{n}(\psi)\right)\right\| \rightarrow 0
$$


as $n \rightarrow \infty$. Then

$$
\limsup _{n \rightarrow \infty}\left|\Lambda_{n}\right|^{-1} \log Q_{\Lambda_{n}}\left(\exp \left(-H_{n}\right)\right) \leqq-\inf \left[I_{E}+H_{1 \mathrm{sc}}\right]
$$

and

$$
\liminf _{n \rightarrow \infty}\left|A_{n}\right|^{-1} \log Q_{A_{n}}\left(\exp \left(-H_{n}\right) \geqq-\inf \left[I_{E}+H^{\mathrm{usc}}\right]\right.
$$

Suppose in addition that each $H_{n}$ is a function of $L_{n}$ and $H$ satisfies

$$
\inf \left[I_{E}+H_{\text {lsc }}\right]=\inf \left[I_{E}+H^{\text {usc }}\right]<\infty .
$$

Then $\left(Q_{n}^{H}\right)$ is sequentially compact, and each accumulation point of $\left(Q_{n}^{H}\right)$ has the form $\int Q^{y} w(d v)$ with a Borel probability measure $w$ on the compact set $\left\{I_{E}+H_{1 s e}\right.$ $=\min \}$.

\subsection{Application to one-dimensional systems}

We will now specialize our results to the case of space dimension $d=1$. Thus $S=\mathbb{R}$, the real line. We confine ourselves to the case of unmarked particles. Hence $E$ is assumed to be a singleton, so that $X$ can be identified with $S=\mathbb{R}$ and $\Omega^{0}$ with $\Omega$, and $Q$ is the Poisson point process on $\mathbb{R}$ with intensity $z:=\mu(E)$ $>0$. We are interested in the sequence of spacings between the particles. First we recall that, for each $P \in \mathscr{P}_{\Theta}, P^{0}$ is supported on the set

$$
\Omega_{\infty}^{0}=\{\omega \in \Omega: \omega(\{0\})=1, \omega(-\infty, 0)=\omega(0, \infty)=\infty\},
$$

cf. [11]. For each $\omega \in \Omega_{\infty}^{0}$ we let $\sigma(\omega)=\left(\sigma_{i}(\omega)\right)_{i \in \mathbb{Z}}$ denote the sequence of spacings between the particles of $\omega$. By definition,

$$
\sigma_{1}(\omega)=\min \{x>0 ; \omega(\{x\})=1\},
$$

$\sigma_{i+1}(\omega)=\sigma_{1}\left(\vartheta_{\sigma_{1}+\ldots+\ldots+\sigma_{i}} \omega\right)$ for $i \geqq 1$, and similarly for $i \leqq 0$. The mapping $\sigma$ : $\omega \rightarrow \sigma(\omega)$ is a bimeasurable bijection from $\Omega_{\infty}^{0}$ onto the sequence space $\Omega^{\#}=$ $(0, \infty)^{\mathbb{Z}}$ with its usual $\sigma$-algebra [11]. For each $P \in \mathscr{P}_{\Theta}$ we let $P^{\#}=P^{0} \circ \sigma^{-1}$ denote the image of $P^{0}$ under $\sigma$. It is well-known [11] that $P^{\#}$ is invariant under the shift $\tau$ on $\Omega^{\#}$. Hence $P^{\#} \in \mathscr{M}_{\tau}^{\#}$, the set of all finite $\tau$-invariant measures on $\Omega^{\#}$. We equip $\mathscr{M}_{\tau}^{\#}$ with the evaluation $\sigma$-algebra and the topology $\tau_{\mathscr{L} *}$ induced by the class $\mathscr{L}^{\#}$ of all bounded cylinder functions on $\Omega^{*}$.

Remark 3.7 The mapping $\pi^{\#}: \mathscr{P}_{\Theta} \rightarrow \mathscr{M}_{\tau}^{\#}$ defined by $\pi^{\#}(P)=P^{\#}$ is affine, measurable, $\tau_{\mathscr{L}}-\tau_{\mathscr{L}}$-continuous, and injective with image

$$
\pi^{\#}\left(\mathscr{P}_{\Theta}\right)=\left\{\rho \in \mathscr{M}_{\tau}^{\#}: \rho=0 \text { or } \rho\left(r_{1}\right)=1\right\} .
$$

Here $r_{i}: \Omega^{*} \rightarrow(0, \infty)$ is the projection onto the $i$-th coordinate.

Proof. We only prove the continuity of $\pi^{\#}$ because all other assertions are obvious or well-known [11]. So let $h \in \mathscr{L}^{\#}$. By $\tau$-invariance, we may assume that $h$ only depends on the coordinates $1, \ldots, k$. For each $t>0$, the mapping $h_{t}=h \circ \sigma 1_{\left\{\sigma_{1}+\ldots+\sigma_{k} \leqq r\right\}}$ on $\Omega^{0}$ is $\mathscr{F}_{[0, t]}$-measurable and thus belongs to $\mathscr{L}^{0}$. 
Remark 2.2 therefore implies that the mapping $P \rightarrow P^{0}\left(h_{t}\right)$ on $\mathscr{P}_{\Theta}$ is continuous. But

$$
\begin{aligned}
\sup _{P \in \mathscr{P}_{\Theta}}\left|P^{*}(h)-P^{0}\left(h_{t}\right)\right| & \leqq\|h\| \sup _{\rho \in \pi^{*}\left(\mathscr{P}_{\Theta}\right)} \rho\left(r_{1}+\ldots+r_{k}>t\right) \\
& \leqq k\|h\| / t .
\end{aligned}
$$

Hence $P \rightarrow P^{\#}(h)$ is a uniform limit of continuous functions.

For each $n \geqq 1$ and $\omega \in \Omega_{A_{n}}$ we consider the empirical spacing process $R_{n, \omega}^{\#}$ $=\pi^{\#}\left(R_{n, \omega}\right)$. More explicitly, if $\omega=\sum_{i=1}^{k} \delta_{x_{i}}$ with $x_{1}<\ldots<x_{k}$ and $\left(x_{i}\right)_{i \in \mathbb{Z}}$ is the canonical enumeration of the points of $\omega^{(n)}$ then

$$
R_{n, \infty}^{\#}=\left|A_{n}\right|^{-1} \sum_{j=1}^{k} \delta_{\left(x_{i+j+1}-x_{i+j}\right)_{i \in \mathbb{Z}}}
$$

Up to the intensity factor $\omega\left(A_{n}\right) /\left|A_{n}\right|, R_{n, \omega}^{\#}$ thus equals the average of the Dirac measures at the finitely many translates of the periodic sequence of spacings between the particles of $\Omega^{(n)}$. We define a functional $I^{\#}: \mathscr{M}_{\tau}^{\#} \rightarrow[0, \infty]$ by setting $I^{\#}(\rho)=z$ if $\rho=0$,

$$
I^{\#}(\rho)=\rho(1) \lim _{k \rightarrow \infty} \frac{1}{k} I\left(\rho_{\{1, \ldots, k\}} / \rho(1) ; \gamma^{k}\right)
$$

if $\rho(1)>0$ and $\rho\left(r_{1}\right)=1$, and $I^{\#}(\rho)=\infty$ if $\rho \notin \pi^{\#}\left(\mathscr{P}_{\Theta}\right)$. In (3.17), $\gamma$ stands for the exponential distribution on $(0, \infty)$ with parameter $z=\mu(E)$, and $\rho_{\{1, \ldots, k\}}$ $=\rho \circ\left(r_{1}, \ldots, r_{k}\right)^{-1}$. This means that, in the nontrivial case, $I^{\#}(\rho)$ is precisely the (negative) specific entropy of the normalized measure $\rho / \rho(1)$ relative to $\gamma$, times the total mass $\rho(1)$ of $\rho$. The significance of $I^{\#}(\rho)$ comes from the following result of Papangelou [15]; cf. also [2].

Proposition 3.8 For each $P \in \mathscr{P}_{\Theta}, I(P)=I^{\#}\left(P^{\#}\right)$.

(As a matter of fact, this equation is proved in [15] under two hypotheses (I) and (II) which can be shown to hold except when $I(P)=\infty=I^{\#}\left(P^{\#}\right)$. We omit the details.) The following corollary to Theorems 3.1 and 3.3 is now immediate.

Corollary 3.9 In the setting described above, let $G: \mathscr{M}_{\tau}^{\#} \rightarrow(-\infty, \infty]$ be such that $G(\rho) \geqq-b(1+\rho(1))$ for some $b<\infty$ and, for each $n \geqq 1, G_{n}: \Omega_{\Lambda_{n}} \rightarrow(-\infty, \infty]$ a measurable function such that

$$
\left\|\left(\left|A_{n}\right|^{-1} G_{n}-G\left(R_{n}^{\#}\right)\right) /\left(1+\left|A_{n}\right|^{-1} N_{A_{n}}\right)\right\| \rightarrow 0
$$

as $n \rightarrow \infty$. Then

$$
\limsup _{n \rightarrow \infty}\left|A_{n}\right|^{-1} \log Q_{A_{n}}\left(e^{-G_{n}}\right) \leqq-\inf \left[I^{\#}+G_{1 s \mathfrak{c}}\right]
$$

and

$$
\liminf _{n \rightarrow \infty}\left|A_{n}\right|^{-1} \log Q_{\Lambda_{n}}\left(e^{-G_{n}}\right) \geqq-\inf \left[I^{\#}+G^{\text {usc }}\right] .
$$


If, in addition, each $G_{n}$ is a function of $R_{n}$ and the right sides of the preceding inequalities are equal and finite then $\left(Q_{n}^{G}\right)$ is sequentially compact and each accumulation point of $\left(Q_{n}^{G}\right)$ has the form $\int P_{\rho} w(d \rho)$ with a Borel probability measure $w$ on the compact set $\left\{I^{\#}+G_{\mathrm{lsc}}=\min \right\}$. Here $P_{\rho}$ is the unique element of $\mathscr{P}_{\Theta}$ with $\pi^{\#}\left(P_{\rho}\right)=\rho$.

\section{Examples}

This section contains some typical applications of the results above. In the first three examples, the underlying asymptotic empirical functionals only depend on the empirical mark intensity measures $L_{n}$; so these examples refer to Corollary 3.6. The subsequent examples are devoted to the more interesting case of particle systems with a position-dependent interaction which can be expressed in terms of the individual empirical fields $R_{n}^{0}$.

Example 4.1 The ideal gas. Here we consider Example (2) of Sect. 2.1. That is, we set $E=\mathbb{R}^{d}$ and think of the elements $v \in E$ as the possible velocities of the particles. The a priori mark distribution $\mu$ is the standard Maxwellian velocity distribution, i.e., $\mu(d v)=(2 \pi)^{-d / 2} \exp \left[-|v|^{2} / 2\right] d v$. (We refrain from considering the more general case of dispersion $\neq 1$ and intensity $\neq 1$ because this gives no additional insight.) A natural choice for the growth function $\psi$ is $\psi(v)=1+|v|$, $v \in E$. We are going to apply Corollary 3.6 to functionals $H$ on $\mathscr{M}_{E}$ which only depend on the intensity $v(E) \geqq 0$ and the (normalized) mean $m(v)=v(v) / v(E)$ of $v \in \mathscr{M}_{E}$. Here we write $v$ for the identity function on $E$, and we leave $m(v)$ undefined when $v \equiv 0$. Note that for each $n \geqq 1$ and $\omega \in \Omega_{A_{n}}, L_{n, \omega}(E)$ is just the particle density of $\omega$ in $A_{n}$, whereas $m\left(L_{n, \omega}\right)$ is the average velocity (=the velocity of the barycenter) of the particles in $\omega$. We consider the events

$$
A_{n}=\left\{\omega \in \Omega_{A_{n}}: a<L_{n, \omega}(E)<b,\left|m\left(L_{n, \omega}\right)\right|>c\right\},
$$

where $0 \leqq a<b \leqq \infty$ and $0 \leqq c<\infty$. These correspond to the set

$$
K=\left\{v \in \mathscr{M}_{E}: a<v(E)<b,|m(v)|>c\right\} .
$$

In view of our choice of $\psi$, we have $1 \in \mathscr{L}_{E}$ and $v \in \mathscr{L}_{E}^{d}$. Hence $K$ is $\tau_{E^{-}}$open, and its closure is obtained by admitting equality in the inequalities defining $K$.

Let $\mu_{m}$ be the Gauss distribution on $E$ with mean $m \in \mathbb{R}^{d}$ and standard covariance. Following the strategy of the proof of Proposition 3.5 it is easy to show that, for each $q \geqq 0, q \mu_{m}$ is the unique measure minimizing $I_{E}$ among all $v \in \mathscr{H}_{E}$ with $v(E)=q$ and $m(v)=m$, and this minimum is given by $J(q,|m|)$, where

$$
J(q, r)=1-q+q \log q+q r^{2} / 2, \quad q, r \geqq 0 .
$$

We thus can conclude that (3.15) holds for $H=\infty 1_{K^{c}}$, and Corollary 1.6 yields that

$$
\lim _{n \rightarrow \infty}\left|A_{n}\right|^{-1} \log Q_{A_{n}}\left(A_{n}\right)=-J\left(q^{*}, c\right)
$$

and

$$
\tau_{\mathscr{L}}-\lim _{n \rightarrow \infty} Q_{A_{n}}\left(\cdot \mid A_{n}\right)=Q^{*}:=\int Q^{q^{*} \mu_{m}} \rho_{c}(d m) .
$$


Here $q^{*}=\left(e^{-c^{2} / 2} \vee a\right) \wedge b$, and $\rho_{c}$ stands for the normalized surface measure on the sphere $\{|\cdot|=c\}$. (For the second assertion we use the fact that the conditional distributions $Q_{A_{n}}\left(\cdot \mid A_{n}\right)$ are invariant under simultaneous rotation of all velocities.) It is no surprise that the limiting measure $Q^{*}$ is a mixture of Poisson point random fields with Maxwellian velocity distributions with means of modulus $c$, but is less obvious and interesting to note that the intensity $q^{*}=z\left(Q^{*}\right)$ depends on the choice of $c$. This shows that the condition of untypically large average velocities favours small particle numbers.

Example 4.2 A Curie-Weiss gas model. Here we let $E$ be the unit sphere in $\mathbb{R}^{d}$, and the elements $s \in E$ are thought of as the possible spins of the particles. We let $\mu$ denote the normalized surface measure (resp., for $d=1$, the equidistribution) on $E$, and we set $\psi=1$. We are interested in the functional $H$ on $\mathscr{M}_{E}$ defined by

$$
H(v)=-|v(\sigma)|^{2} / 2 v(E) \quad\left(v \in \mathscr{M}_{E} \backslash\{0\}\right),
$$

where $\sigma: E \rightarrow E$ stands for the identity map. For $v \equiv 0$ we set $H(v)=0 . H$ is continuous and satisfies $H(v) \geqq-v(E) / 2$ for all $v \in \mathscr{M}_{E}$. For each $n \geqq 1$ and $\omega \in \Omega_{A_{n}}$ we define $H_{n}(\omega)=\left|A_{n}\right| H\left(L_{n, \omega}\right)$. More explicitly, if $\omega=\sum_{x \in \xi} \delta_{\left(x, s_{x}\right)}$ with a finite non-
empty $\xi \subset A_{n}$ and $s_{x} \in E$ then

$$
H_{n}(\omega)=-(\operatorname{card} \xi)^{-1} \frac{1}{2} \sum_{x, y \in \xi} s_{x} \cdot s_{y} .
$$

Thus $H_{n}$ is the Hamiltonian of a system of spinning particles in $A_{n}$ where any two particles $x, y \in \xi$ interact with an exchange energy $-(\text { card } \xi)^{-1} s_{x} \cdot s_{y}$. We will apply Corollary 3.6 to the functions $\left\{\left(\beta H_{n}\right), \beta H\right\}$, where $\beta>0$ is a temperature parameter. So we need to determine $\min \left[I_{E}+\beta H\right]$ and the measures on $E$ for which this minimum is attained. To this end we introduce the probability measures

$$
\mu_{h}=e^{h \cdot \sigma} \mu / \mu\left(e^{h \cdot \sigma}\right), \quad h \in \mathbb{R}^{d} .
$$

It is easy to see that for each $m \in \mathbb{R}^{d}$ with $|m|<1$ there exists some $h=h(m) \in \mathbb{R}^{d}$ with $\mu_{h}(\sigma)=m$. Now let $v \in \mathscr{M}_{E}$ and suppose $v$ is not supported on a single point. Writing $q=v(E)$, we can find an $h \in \mathbb{R}^{d}$ such that $v(\sigma)=q \mu_{h}(\sigma)$. A straightforward computation yields

$$
I_{E}(v)+\beta H(v)=I\left(v ; q \mu_{h}\right)+J_{\beta}(q,|h|),
$$

where $J_{\beta}$ is defined as follows. Let $\sigma_{1}: E \rightarrow[-1,1]$ denote the projection on the first coordinate and set $\varphi(r)=\log \mu\left(e^{r \sigma_{1}}\right), r \in \mathbb{R}$. By rotational invariance of $\mu$ we have $\left|\mu_{h}(\sigma)\right|=\varphi^{\prime}(|h|)$ for all $h \in \mathbb{R}^{d}$. Now $J_{\beta}:[0, \infty)^{2} \rightarrow[0, \infty)$ is given by

$$
J_{\beta}(q, r)=1-q+q \log q+q \Phi_{\beta}(r)
$$

with $\Phi_{\beta}(r)=r \varphi^{\prime}(r)-\beta \varphi^{\prime}(r)^{2} / 2-\varphi(r)$.

From (4.1) we conclude that $\inf \left[I_{E}+\beta H\right]=\inf J_{\beta}$ and $\left\{I_{E}+\beta H=\min \right\}$ $=\left\{q \mu_{h}: J_{\beta}(q,|h|)=\min \right\}$. To find the minimal points of $J_{\beta}$ we first seek those of $\Phi_{\beta}$. Since $\Phi_{\beta}^{\prime}(r)=\varphi^{\prime \prime}(r)\left[r-\beta \varphi^{\prime}(r)\right]$ and $\varphi^{\prime \prime}(r)$ is the variance of $\sigma_{1}$ relative to $\mu_{(r, 0, \ldots, 0)}, \Phi_{\beta}^{\prime}(r)$ can only vanish when $\varphi^{\prime}(r)=r / \beta$. This equation always admits the solution $r=0$. If $d=1$ or $d \geqq 3$, a result of Ellis and Newman [4] shows that $\varphi^{\prime}$ is concave on $[0, \infty)$. Since $\varphi^{(4)}(0)=\mu\left(\sigma_{1}^{4}\right)-3 \mu\left(\sigma_{1}^{2}\right)^{2}<0$, this implies that 
the function $r \rightarrow \varphi^{\prime}(r) / r$ is strictly decreasing on $[0, \infty)$. In the case $d=2$, the same conclusion can be derived by means of an explicit expansion of $e^{\varphi}$. Consequently, if $\varphi^{\prime \prime}(0) \leqq 1 / \beta$ then $\Phi_{\beta}^{\prime}(r)=0$ only when $r=0$, whereas in the case $\varphi^{\prime \prime}(0)$ $>1 / \beta$ there exists a unique $r^{*}=r(\beta)>0$ such that $\varphi^{\prime}\left(r^{*}\right)=r^{*} / \beta$ and thereby $\Phi_{\beta}\left(r^{*}\right)<0=\Phi_{\beta}(0)$. Noticing further that $\varphi^{\prime \prime}(0)=\mu\left(\sigma_{1}^{2}\right)=\mu\left(|\sigma|^{2}\right) / d=1 / d$ we end up with the following conclusion:

For $0 \leqq \beta \leqq d, \Phi_{\beta}$ attains its minimum 0 precisely at $r=0$, whence $J_{\beta}$ reaches its minimum 0 exactly when $q=1, r=0$. In the case $\beta>d, \Phi_{\beta}$ has a negative minimum which is attained at $r^{*}$ only, and $\left\{J_{\beta}=\min \right\}=\left\{\left(q^{*}, r^{*}\right)\right\}$, where $q^{*}=$ $q(\beta):=\exp \left[-\Phi_{\beta}\left(r^{*}\right)\right]>1$. Corollary 3.6 thus implies that

$$
\lim _{n \rightarrow \infty}\left|\Lambda_{n}\right|^{-1} \log Q_{A_{n}}\left(e^{-\beta H_{n}}\right)= \begin{cases}0 & \text { if } 0<\beta \leqq d, \\ q^{*}-1 & \text { if } \beta>d,\end{cases}
$$

and

$$
\lim _{n \rightarrow \infty} Q_{n}^{\beta H}= \begin{cases}Q & \text { for } 0 \leqq \beta \leqq d, \\ Q^{*}:=\int Q^{q^{*} \mu_{h}} \rho_{r^{*}}(d h) & \text { for } \beta>d .\end{cases}
$$

Here $\rho_{r^{*}}$ stands for the normalized surface measure on the sphere $\left\{h \in \mathbb{R}^{d}\right.$ : $\left.|h|=r^{*}\right\}$, and we have used that $Q_{n}^{\beta H}$ is invariant under simultaneous rotation of all spins. Hence, at the critical point $\beta=d$ there is not only a transition of the average magnetization of typical configurations from 0 to the modulus $m(\beta)=\varphi^{\prime}(r(\beta))>0$, but also a change of the particle density from 1 to $q(\beta)>1$. To conclude, we remark that the behaviour of $q(\beta)$ and $m(\beta)$ near the critical point is given by

$$
q(\beta)-1 \sim \frac{d+2}{4 d^{2}}(\beta-d)^{2} \quad \text { and } \quad m(\beta) \sim d^{-1}(d+2)^{1 / 2}(\beta-d)^{1 / 2} \text { as } \beta \downarrow d .
$$

We also note that the results above can easily be extended to the case when the spin space $E$ equals the whole $\mathbb{R}^{d}$ and $\mu$ is suitably chosen.

Example 4.3 Brownian particles with constraints on the displacement of their barycenter. In this example we set $E=\left\{\zeta \in \mathscr{C}\left([0,1], \mathbb{R}^{d}\right): \zeta(0)=0\right\}$. Each $\zeta \in E$ is interpreted as a possible time evolution of a particle; that is, a particle at position $x$ with mark $\zeta$ is considered to move along the path $(x+\zeta(t))_{0 \leqq t \leqq 1}$ during the time interval $[0,1] . E$ is equipped with the uniform metric and the Wiener measure $\mu$ on the associated Borel $\sigma$-algebra $\mathscr{B}_{E}$. We choose the growth function $\psi(\zeta)=1+\|\zeta\|$, where $\|\cdot\|$ is the sup-norm. By abuse of notation, we also use the symbol $\zeta(t)$ to denote the projection mapping $\zeta \rightarrow \zeta(t)$ on $E, 0 \leqq t \leqq 1$. We consider the expected path $\eta(v)=(v(\zeta(t)) / v(E))_{0 \leqq t \leqq 1}$ of any $v \in \mathscr{M}_{E}, v \neq 0$. Since $|\zeta(t)| \leqq \psi$ for all $t$, the dominated convergence theorem shows that $\eta(v) \in E$ for all $0 \neq v \in \mathscr{M}_{E}$. Moreover, it is easily seen that the mapping $v \rightarrow \eta(v)$ on $\mathscr{M}_{E} \backslash\{0\}$ becomes continuous if $E$ is equipped with the topology of pointwise convergence. Let $\mathscr{U} \subset E$ be open in this topology and define

$$
A_{n}=\left\{N_{A_{n}} \geqq 1, \eta\left(L_{n}\right) \in \mathscr{U}\right\} .
$$

Note that for each $\omega=\sum_{x \in \xi} \delta_{\left(x, \zeta_{x}\right)} \in \Omega_{A_{n}}$

$$
\eta\left(L_{n, \omega}\right)=\left((\operatorname{card} \xi)^{-1} \sum_{x \in \xi} \zeta_{x}(t)\right)_{0 \leqq t \leqq 1}
$$


describes the path of displacements of the gravicenter of the particles in $\omega$. So $A_{n}$ is the event that this path belongs to the prescribed set $\mathscr{U}$. To apply Corollary 3.6 we recall that, for each $\eta \in E, I_{E}$ takes a finite value on $\left\{v \in \mathscr{M}_{E}\right.$ : $v(E)=1, \eta(v)=\eta\}$ if and only if $\eta$ is absolutely continuous, and in this case we have

$$
\min I_{E}\left(\left\{v \in \mathscr{M}_{E}: v(E)=1, \eta(v)=\eta\right\}\right)=J(\eta):=\frac{1}{2} \int_{0}^{1}|\dot{\eta}(t)|^{2} d t
$$

and the unique measure attaining this minimum is $\mu_{\eta}$, the Wiener measure with mean $\eta$, i.e., with deterministic drift $\dot{\eta}$. This is a well-known fact in connection with Schilder's theorem on large deviations of Brownian motion; see, for example, [5]. Using the remark below (2.11) we conclude that

$$
\min I_{E}\left(\left\{v \in \mathscr{M}_{E}: v=0 \text { or } \eta(v)=\eta\right\}\right)=1-e^{-J(\eta)}
$$

with unique minimizing measure $v=e^{-J(\eta)} \mu_{\eta}$, provided $\eta \in E$ is absolutely continuous. Suppose now $\mathscr{U}$ is convex and contains an absolutely continuous $\eta$ with $J(\eta)<\infty$. Then $c^{*}:=\min J(\operatorname{cl} \mathscr{U})=\inf J(\mathscr{U})<\infty$, and there exists a unique $\eta^{*} \in \operatorname{cl} \mathscr{U}$ with $J\left(\eta^{*}\right)=c^{*}$. Setting $\mu^{*}=e^{-c^{*}} \mu_{\eta^{*}}$ we thus obtain from Corollary 3.6 that

$$
\tau_{\mathscr{L}}-\lim _{n \rightarrow \infty} Q_{\Lambda_{n}}\left(\cdot \mid A_{n}\right)=Q^{\mu^{*}}
$$

We note again that the condition of an untypical displacement of the barycenter leads to a lowering of the intensity from the a priori value 1 to the value $e^{-c^{*}}$.

So far we only considered functionals of the empirical mark distribution $L_{n}$ which contains no information on the position of the particles. We now turn to the more general case of functionals of the individual empirical fields $R_{n}^{0}$. Namely, we consider asymptotic empirical functionals $\left\{\left(G_{n}\right), G\right\}$ with a functional $G$ of the form $G=e_{g} \circ \pi^{0}: P \rightarrow P^{0}(g)$ on $\mathscr{P}_{\Theta}$, where $g:(u, \omega) \rightarrow g(u, \omega)$ is a function on $\Omega^{0}$ which should be interpreted as the contribution of a particle at the origin of type $u$ to the total energy of all particles of $\omega$. Formally, this includes the case when $G_{n}$ is the Hamiltonian (with periodic boundary condition) relative to a translation invariant pair interaction. Unfortunately, the associated function $g$ fails to be bounded even if the pair interaction is bounded with bounded support, so that this important case does not fit directly into our setting. Fortunately, it is not too difficult to adapt our techniques to the case of hard core interactions [9], and using some ideas of [14] one can also treat the case of superstable interactions. Here we will discuss two other interesting types of interaction which are covered by the results of this paper.

Example 4.4 The Widom-Rowlinson model. This is a model of possibly penetrating balls whose interaction depends on their degree of penetration. Let $B$ denote the unit ball (or any other bounded figure) in $\mathbb{R}^{d}$ and $E=[0, q]$ for some $q>0$. A particle at position $x$ with mark $r \in E$ is visualized as the ball $x+r B$ with center $x$ and radius $r$. We set $\psi=1$ and let $\mu$ be any finite measure on $E$. For each $n \geqq 1$ and $\omega \in \Omega_{\Lambda_{n}}$ we consider the expression

$$
G_{n}(\omega)=\left|\bigcup_{(x, r) \in \omega}(x+r B)_{n}\right|-\sum_{(x, r) \in \omega}\left|(x+r B)_{n}\right|
$$


which measures the degree of overlap of the periodized balls

$$
(x+r B)_{n}=\left\{y \in A_{n}: y+i \in X+r B \text { for some } i \in p_{n} \mathbb{Z}^{d}\right\}
$$

with $(x, r) \in \omega$. Here we have identified $\omega$ with its support. $G_{n}(\omega)$ is the Hamiltonian of Widom and Rowlinson [20] in $A_{n}$ with periodic boundary condition. As we will see below, the one-particle contribution to $G_{n}(\omega)$ is given by the function

$$
g(r, \omega)=-\int_{r B}\left[1-\omega(B(y))^{-1}\right] d y,
$$

where $(r, \omega) \in \Omega^{0}$ and $B(y)=\{(x, r) \in S \times E: y \in x+r B\}$ corresponds to the set of all balls containing $y$. We note first that $\omega(B(y)) \geqq 1$ whenever $(0, r) \in \omega$ and $y \in r B$. Hence $0 \geqq g \geqq-q^{d}|B|$ and thereby $g \in \mathscr{L}^{0}$. The associated functional $G: P \rightarrow P^{0}(g)$ on $\mathscr{P}_{\theta}$ is therefore continuous. Using (2.3) and the reflection invariance of Lebesgue measure, we see that this functional is given by

$$
\begin{aligned}
G(P) & =-\iint 1_{r B}(-x)\left[1-\omega(B(-x))^{-1}\right] d x P^{0}(d r, d \omega) \\
& =-\iint 1_{x+r B}(0)\left[1-\omega(B(0))^{-1}\right] \omega(d x, d r) P(d \omega) \\
& =-P\left(1_{\{N(B(0)) \geqq 1\}}[N(B(0))-1]\right) \\
& =P(N(B(0)) \geqq 1)-P(N(B(0))) .
\end{aligned}
$$

In particular, for each $n \geqq 1$ and $\omega \in \Omega_{A_{n}}$ we find

$$
\left|A_{n}\right| G\left(R_{n, \omega}\right)=\int_{A_{n}}\left[1_{\left\{\vartheta_{y} \omega(n)(B(0)) \geqq 1\right\}}-\vartheta_{y} \omega^{(n)}(B(0))\right] d y=G_{n}(\omega) .
$$

We consider the asymptotic empirical functionals $\left\{\left(\beta G_{n}\right), \beta G\right\}$, where $\beta>0$ is a temperature parameter. (Note that Widom-Rowlinson Hamiltonians with other than periodic boundary conditions would also satisfy (3.1).) First, Corollary 3.2 asserts that the "pressure"

$$
p(\beta)=\lim _{n \rightarrow \infty}\left|\Lambda_{n}\right|^{-1} \log Q_{A_{n}}\left(e^{-\beta G_{n}}\right)
$$

exists and equals $-\inf \left[I^{0}+\beta e_{g}\right]$. This is usually called the Gibbs variational formula. Using the extension of Theorem 3.1 which was mentioned around (3.5), we obtain further that the distribution of $R_{n}$ under the periodic Gibbs distribution $Q_{n}^{\beta G}$ satisfies a large deviation principle with rate function $I^{\beta G}=I+\beta G$ $+p(\beta)$. Finally, Corollary 3.4 shows that each accumulation point of the sequence $\left(Q_{n}^{\beta G}\right)$ belongs to the convex compact set $M^{\beta G}=\left\{I^{\beta G}=0\right\} . M^{\beta G}$ coincides with the set of all Gibbs measures for the Widom-Rowlinson interaction at inverse temperature $\beta$. To see this one might identify $\Omega$ with $\left(\Omega_{[0,1)^{d}}\right)^{\mathbb{Z}^{d}}$ by subdividing $S$ into disjoint unit cells, and then apply the variational principle of Lanford and Ruelle, cf. Theorem 15.39 of [7]. In particular, if $d=1$ or $\beta$ is small we may conclude from Theorems 8.39 and 8.7 of [7] that $M^{\beta G}$ contains a unique element $P_{\beta}$. Thus in this case $Q_{n}^{\beta G} \rightarrow P_{\beta}$ as $n \rightarrow \infty$, and $P_{\beta}$ is also the limit of microcanonical Gibbs distributions; cf. the discussion in Sect. 2.3 of [8]. On the other hand, it is known $[18,20]$ that $M^{\beta G}$ is not a singleton when $d \geqq 2, \beta$ is large, and $\mu=z \delta_{q}$ for some specific large number $z=z(\beta)>0$. This is the reason for the physical interest in this model. 
Example 4.5 One-dimensional systems with nearest-particle interaction. Here we consider the setting of Sect. 3.4, i.e., $d=1$ and $E$ is a singleton. We fix any bounded measurable function $\varphi:(0, \infty) \rightarrow \mathbb{R}$ (which is not constant Lebesguealmost everywhere) and look at the functions

$$
G_{n}=\left|A_{n}\right| R_{n}^{0}\left(\varphi \circ \sigma_{1}\right)=\left|A_{n}\right| R_{n}^{\#}\left(\varphi \circ r_{1}\right)
$$

on $\Omega_{\Lambda_{n}}, n \geqq 1$. For each $n$ and $\omega=\sum_{i=1}^{k} \delta_{x_{i}} \in \Omega_{\Lambda_{n}}$ with $x_{1}<\ldots<x_{k}$ we have

$$
G_{n}(\omega)=\sum_{i=1}^{k-1} \varphi\left(x_{i+1}-x_{i}\right)+\varphi\left(x_{1}+p_{n}-x_{k}\right)
$$

That is, $G_{n}$ is the Hamiltonian in $A_{n}$ with periodic boundary condition for the interaction potential $\varphi$ between nearest particles. We are interested in the events

$$
A_{n}=\left\{\omega \in \Omega_{\Lambda_{n}}: G_{n}(\omega)>a \omega\left(A_{n}\right)\right\}
$$

that the interaction energy per particle exceeds a given number $a$. Clearly, $A_{n}=$ $\left\{R_{n}^{\#} \in K_{a}\right\}$, where

$$
K_{a}=\left\{\rho \in \mathscr{M}_{\tau}^{\#}: \rho\left(\varphi \circ r_{1}\right)>a \rho(1)\right\}
$$

$K_{a}$ is convex and $\tau_{\mathscr{L}}$-open. To apply Corollary 3.9 we note first that

$$
\begin{aligned}
\inf _{\rho \in K_{\boldsymbol{a}}} I^{\#}(\rho) & =\inf \left\{\rho_{1}(1) I\left(\rho_{1} / \rho_{1}(1) ; \gamma\right): \rho_{1}(r)=1, \rho_{1}(\varphi)>a \rho_{1}(1)\right\} \\
& =\inf \left\{\alpha(r)^{-1} I(\alpha ; \gamma): \alpha(\varphi)>a\right\} .
\end{aligned}
$$

Here $r$ stands for the identity map on $(0, \infty)$, the first infimum extends over all finite measures $\rho_{1}$ on $(0, \infty)$ which are the one-dimensional marginal of some $\rho \in K_{a} \cap\left\{I^{\#}<\infty\right\}$ and the second over all probability measures $\alpha$ on $(0, \infty)$ with the stated properties. (Note that $\alpha(r)<\infty$ whenever $I(\alpha ; \gamma)<\infty$.) To identify the second infimum we introduce the function

$$
c(b)=\inf \{I(\alpha ; \gamma): \alpha(\bar{\varphi})=b\},
$$

where $\bar{\varphi}=(\varphi, r), b \in \mathbb{R}^{2}$, and the inf extends over all probability measures $\alpha$ on $(0, \infty)$ with $\alpha(\bar{\varphi})=b$. Clearly, $c(b) \geqq 0$ with equality precisely for $b=\gamma(\bar{\varphi})=(\gamma(\varphi), 1 / z)$. It is well-known that

$$
c(b)=\sup _{t \in \mathbb{R}^{2}}\left[t \cdot b-\log \gamma\left(e^{t \cdot \bar{\varphi}}\right)\right],
$$

and $c$ is essentially strictly convex and essentially smooth, cf. [16]. Moreover, for each $b \in \operatorname{int}\{c<\infty\}$ the supremum in (4.5) is reached precisely for $t=\operatorname{grad} c(b)$, and the associated measure $\gamma_{t}=e^{t \cdot \bar{\varphi}} \gamma / \gamma\left(e^{t \cdot \bar{\varphi}}\right)$ is the unique $\alpha$ attaining the infimum in (4.4). Finally, if $a \in(\operatorname{ess}-i n f \varphi$, ess-sup $\varphi)$ then there exists some $m>0$ with $(a, m) \in \operatorname{int}\{c<\infty\}$, and thus a unique $m_{a}>0$ which minimizes the function $m \rightarrow c(a, m) / m$. Also, using the essential strict convexity of $c$ and the fact that $c\left(\gamma(\varphi), z^{-1}\right)=0$ one readily sees that the function $J: a \rightarrow c\left(a, m_{a}\right) / m_{a}$ is strictly 
increasing on $(\gamma(\varphi)$, ess-sup $\varphi)$, and the essential smoothness of $c$ implies that $J$ is continuous. It is also easy to check that $J(a)<z$ for all these $a$. Combining all these facts we can conclude that

$$
\inf _{\rho \in K_{a}} I^{\#}(\rho)=\min _{\rho \in \mathrm{cl} K_{a}} I^{\#}(\rho)=c\left(a, m_{a}\right) / m_{a}=J(a),
$$

and the unique measure $\rho \in \mathrm{cl} K_{a}$ minimizing $I^{\#}$ is $\rho=m_{a}^{-1}\left(\gamma_{\operatorname{grad} c\left(a, m_{a}\right.}\right){ }^{\mathbb{Z}}$. Corollary 3.9 thus gives the following result: If $\gamma(\varphi)<a<\operatorname{ess-sup} \varphi$ and $A_{n}$ is defined by (4.3) then

$$
\lim _{n \rightarrow \infty}\left|A_{n}\right|^{-1} \log Q_{A_{n}}\left(A_{n}\right)=-J(a)
$$

and

$$
\lim _{n \rightarrow \infty} Q_{\Lambda_{n}}\left(\cdot \mid A_{n}\right)=P_{a},
$$

where $P_{a} \in \mathscr{P}_{\Theta}$ is the stationary renewal process with spacing distribution $\gamma_{\operatorname{grad} c\left(a, m_{a}\right)}$. Note that the second component of $\operatorname{grad} c\left(a, m_{a}\right)$ equals $J(a)>0$, whence $m_{a}$ is larger than the value of $m$ which minimizes $c(a, \cdot)$. This means that the spacing distribution of $P_{a}$ is not the distribution $\alpha$ which minimizes $I(\alpha ; \gamma)$ under the constraint $\alpha(\varphi) \geqq a$, but a distribution with a larger expectation. In other words, the intensity of $P_{a}$ is smaller than one might expect at a first sight.

The preceding example is only one of the simplest applications of Corollary 3.9. More generally, one can consider conditional probabilities involving functions of two subsequent spacings, and show that these converge to Wold processes (with a Markovian distribution of spacings). Even more generally, it is also clear how point processes with a Gibbsian distribution of spacings appear as limits of suitable conditional Poisson distributions.

\section{Proofs}

\subsection{Equiintegrability of tame local functions}

Here we deal with some problems which arise from the fact that the class $\mathscr{L}$ defining the topology $\tau_{\mathscr{L}}$ on $\mathscr{P}_{\Theta}$ contains all tame, rather than only all bounded, local functions. (Recall that it is this choice of $\mathscr{L}$ which makes the Palm mapping $\pi^{0}: P \rightarrow P^{0}$ continuous.) Namely, we will show that, for each $\Delta \in \mathscr{C}$ and $c>0$, the function $N_{\Delta}^{\psi}$ is equiintegrable relative to all $P$ in the level set $\{I \leqq c\}$. In particular, this will give us the compactness of $\{I \leqq c\}$ in the topology $\tau_{\mathscr{L}}$. We shall use the following easily verified properties of the Poisson random field $Q$.

Lemma 5.1 For all bounded $\Delta \in \mathscr{B}_{S}$ and measurable functions $h: E \rightarrow[0, \infty)$,

$$
Q\left(\exp \left[\frac{1}{2} N_{4} \log N_{A}\right]\right)<\infty
$$

and

$$
Q\left(\exp N_{A}(h)\right)=\exp \left[|\Delta| \mu\left(e^{h}-1\right)\right] .
$$

Here is the above-mentioned result on the equiintegrability of $N_{4}^{\psi}$. 
Lemma 5.2 For each $c \geqq 0, \varepsilon>0$, and bounded $\Delta \in \mathscr{B}_{S}$ there exists some $b=b(c, \varepsilon, \Delta)<\infty$ such that for all $P \in\{I \leqq c\}$

$$
P\left(N_{A}^{\psi} 1_{\left\{N_{A 1}^{\psi}>b\right\}}\right)<\varepsilon \text {. }
$$

Proof. Let $c, \varepsilon, \Delta$ be given and $P \in\{I \leqq c\}$ arbitrary. We set $a=4|\Delta| c / \varepsilon$. In view of assumption (2.1) and the dominated convergence theorem, there exists a number $s>0$ such that

$$
\delta:=\mu\left(\exp \left[a \psi 1_{\{\psi>s\}}\right]-1\right)<\varepsilon a / 4|\Delta| .
$$

From formulas (2.11) and (2.13) we can conclude that for each $r_{\Delta}^{-1} \mathscr{F}_{\Delta}$-measurable function $f \geqq 0$

$$
P(f)-\log Q\left(e^{f}\right) \leqq I\left(P_{\Delta} ; Q_{\Delta}\right) \leqq|\Delta| c .
$$

Applying this to $f=a N_{\Delta}\left(\psi 1_{\{\psi>s\}}\right)$ and using (5.2) we obtain that

$$
P\left(N_{\Delta}\left(\psi 1_{\{\psi>s\}}\right)\right) \leqq|\Delta| c / a+|\Delta| \delta / a<\varepsilon / 2 .
$$

Setting $f=\frac{1}{2} N_{\Delta} \log N_{\Delta}$ in (5.3) and using (5.1) we find further that

$$
\begin{aligned}
P\left(N_{A} \log N_{A}\right) & \leqq 2|\Delta| c+2 \log Q\left(\exp \left[\frac{1}{2} N_{A} \log N_{A}\right]\right) \\
& =: c_{1}<\infty .
\end{aligned}
$$

Hence, for sufficiently large $n$ we have

$$
P\left(N_{\Delta} 1_{\left\{N_{\Delta}>n\right\}}\right) \leqq c_{1} / \log n<\varepsilon / 4 s .
$$

Observe now that

$$
N_{\Delta}^{\psi} \leqq s N_{\Delta}+N_{\Delta}\left(\psi 1_{\{\psi>s\}}\right)
$$

We thus can write

$$
\begin{aligned}
P\left(N_{\Delta}^{\psi} 1_{\left\{N_{\Delta}^{\psi}>b\right\}}\right) & \leqq s P\left(N_{\Delta} 1_{\left\{N_{\Delta}^{\psi}>b\right\}}\right)+P\left(N_{\Delta}\left(\psi 1_{\{\psi>s\}}\right)\right) \\
& <s P\left(N_{\Delta} 1_{\left\{N_{\Delta}>n\right\}}\right)+\operatorname{sn} P\left(N_{\Delta}^{\psi}>b\right)+\varepsilon / 2 \\
& <3 \varepsilon / 4+\operatorname{sn} P\left(N_{\Delta}^{\psi}\right) / b .
\end{aligned}
$$

On the other hand,

$$
P\left(N_{4}^{\psi}\right) \leqq s P\left(N_{4}\right)+\varepsilon / 2 \leqq s n+3 \varepsilon / 4=: c_{2} .
$$

The lemma thus follows by choosing $b$ so large that $s n c_{2} / b<\varepsilon / 4$.

The lemma above is an essential ingredient of our arguments. Here we will use it to show that the level sets of $I$ are compact in our topology $\tau_{\mathscr{L}}$.

Proof of Proposition 2.6 It follows immediately from (2.13) and (2.11) that $I$ is lower semicontinuous. Hence, its level sets are closed. To prove their compactness we let $\mathscr{L}^{b}$ denote the space of all bounded Iocal functions and $\tau_{\mathscr{L} b}$ the associated topology on $\mathscr{P}_{\Theta}$. By the same arguments as in Propositions 15.14, 4.9 and 4.15 of [7] it follows that the level sets of $I$ are compact and sequentially 
compact in $\tau_{\mathscr{L} b}$. (Note that any additive set function on the algebra $\bigcup_{A \in \mathscr{G}} r_{A}^{-1} \mathscr{F}_{A}$ which is $\sigma$-additive on each $r_{A}^{-1} \mathscr{F}_{A}$ admits a unique extension to a measure on $\mathscr{F}$ because each $\left(\Omega_{A}, \mathscr{F}_{A}\right)$ is standard Borel.) It is thus sufficient to show that the topologies $\tau_{\mathscr{L} b}$ and $\tau_{\mathscr{L}}$ coincide on $\{I \leqq c\}$ for any $c \geqq 0$. So let $f \in \mathscr{L}$ and $\Delta \in \mathscr{C}$ be such that $|f| \leqq b\left(1+N_{d}^{\psi}\right)$ for some $b<\infty$. For each $n \in \mathbb{N}$, the function $f_{n}=f 1_{\left\{N_{\Delta}^{\psi} \leq n\right\}}$ then belongs to $\mathscr{L}^{b}$, and Lemma 5.2 shows that $e_{f_{n}}$ converges to $e_{f}$ uniformly on $\{I \leqq c\}$ as $n \rightarrow \infty$. Hence $e_{f}$ is $\tau_{\mathscr{L} b}$-continuous on $\{I \leqq c\}$, and this implies that $\tau_{\mathscr{L}}=\tau_{\mathscr{L} b}$ on $\{I \leqq c\}$.

\subsection{Comparison of different Poisson random fields}

In this subsection we study the relationship between $Q$ and $Q^{v}$ for an arbitrary $v \in \mathscr{M}_{E}$. In particular, we shall prove Proposition 3.5. The basic fact is stated in the lemma below.

Lemma 5.3 Suppose $v \in \mathscr{M}_{E}$ is $\mu$-continuous with density $h$. Then, for each bounded $\Delta \in \mathscr{B}_{S}, Q_{\Delta}^{v}$ is $Q_{\Delta}$-continuous with density

$$
f_{\Delta}^{v}=\exp \left[N_{\Delta}(\log h)+|\Delta|(\mu(E)-v(E))\right] .
$$

Here we use the convention that $f_{\Delta}^{v}=0$ on the set $\{N(\Delta \times\{h=0\})>0\}$.

Proof. Obvious.

Proof of Proposition 3.5 Let $v \in \mathscr{M}_{E}$ and $P \in \mathscr{P}_{\Theta}$ with $\mu_{P}=v$ be given. To begin, we note that if $I(P)<\infty$ then $v \ll \mu$. Indeed, for each $C \in \mathscr{B}_{S}$ with $|C|=1$ we have $P_{C} \ll Q_{C}$ because $I\left(P_{C} ; Q_{C}\right) \leqq I(P)$. Therefore, if $B \in \mathscr{B}_{E}$ is such that $\mu(B)=0$ then $Q(N(C \times B))=0$ and thus $\nu(B)=\mu_{P}(B)=P(N(C \times B))=0$. Consequently, both sides of (3.12) are infinite except when $\nu \ll \mu$. We thus can assume that $\nu \ll \mu$ with a density $h$.

Let $\Delta \in \mathscr{C}$ be given. By the definition of $f_{\Delta}^{v}$ in (5.4),

$$
\mathrm{P}_{\Delta}\left(f_{\Delta}^{v}>0\right)=P(N(\Delta \times\{h=0\})=0)=1
$$

because $P(N(\Delta \times\{h=0\}))=|\Delta| v(h=0)=0$. Hence $P_{\Delta} \ll Q_{\Delta}$ if and only if $P_{\Delta} \ll Q_{\Delta}^{v}$, and in this case we have

$$
\begin{aligned}
I\left(P_{\Delta} ; Q_{\Delta}\right) & =P_{\Delta}\left(\log d P_{\Delta} / d Q_{\Delta}^{v}+\log f_{A}^{v}\right) \\
& =I\left(P_{\Delta} ; Q_{\Delta}^{v}\right)+\lambda \otimes v\left(1_{\Delta} \otimes \log h\right)+|\Delta|(\mu(E)-v(E)) \\
& =I\left(P_{\Delta} ; Q_{\Delta}^{v}\right)+|\Delta| I_{E}(v) .
\end{aligned}
$$

This identity holds trivially if neither $P_{\Delta} \ll Q_{\Delta}$ nor $P_{\Delta} \ll Q_{\Delta}^{v}$. Dividing by $|\Delta|$ and taking the sup over $\Delta$ we arrive at (3.12). The rest of the proposition is obvious because $I_{v}(P) \geqq 0$ with equality if and only if $P=Q^{v}$. 


\subsection{Large deviations for $R_{n}$}

We will now prove our first main result, Theorem 3.1. So let $\left\{\left(F_{n}\right), F\right\}$ be an asymptotic empirical functional. Our first step consists in showing that there is no loss in assuming that $F \geqq 1+z^{\psi}$ and $F_{n} \geqq 0$ for all $n$.

Lemma 5.4 Let $b \in \mathbb{R}$ and define

$$
F^{*}=F+b\left(1+z^{\psi}\right), \quad F_{n}^{*}=F_{n}+b\left(\left|A_{n}\right|+N_{X_{n}}^{\psi}\right) .
$$

Also, let $Q^{*}=Q^{\mu^{*}}$ be the Poisson point random field with mark intensity measure $\mu^{*}=e^{b \psi} \mu$, and $I^{*}=I_{\mu^{*}}$ the corresponding specific entropy functional. Then $\left\{\left(F_{n}^{*}\right), F^{*}\right\}$ is an asymptotic empirical functional, and inequalities (3.2) and (3.3) hold if and only if they hold for the starred quantities.

Proof. The first claim is obvious. Next, Lemma 5.3 shows that

$$
\left|\Lambda_{n}\right|^{-1} \log Q_{A_{n}}^{*}\left(e^{-F_{n}^{*}}\right)=c+\left|\Lambda_{n}\right|^{-1} \log Q_{\Lambda_{n}}\left(e^{-F_{n}}\right),
$$

where $c=\mu(E)-\mu^{*}(E)-b$. On the other hand, since $I_{\mu_{P}}(P)$ is independent of $Q$ resp. $Q^{*}$ we conclude from (3.12) and (2.10) that

$$
I^{*}=I-c-b\left(1+z^{\psi}\right) .
$$

Since $z^{\psi}$ is continuous, $F_{\mathrm{lsc}}^{*}=F_{\mathrm{lsc}}+b\left(1+z^{\psi}\right)$ and thus $I^{*}+F_{\mathrm{lsc}}^{*}=I+F_{\mathrm{lsc}}-c$, and a similar identity holds for the upper semicontinuous regularization. The lemma is now obvious.

By hypothesis on $\left\{\left(F_{n}\right), F\right\}$, the constant $b$ in Lemma 5.4 can be chosen so large that $F^{*} \geqq 1+z^{\psi}$. We thus can and will assume that $F \geqq 1+z^{\psi}$. We can also assume that $\delta_{n}^{F} \leqq 1$ for all $n$. It then follows from (3.1) that $F_{n} \geqq 0$ for all $n$.

We first derive the upper bound (3.2). We follow the lines of [8] but need to go into the details because of some additional technicalities. We can assume that $Q_{A_{n}}\left(e^{-F_{n}}\right)>0$ for all $n$. The measures $Q_{n}^{F}$ in (3.5) are thus well-defined. By $(2.10)$,

$$
-\left|\Lambda_{n}\right|^{-1} \log Q_{\Lambda_{n}}\left(e^{-F_{n}}\right)=\left|\Lambda_{n}\right|^{-1} I\left(Q_{n}^{F} ; Q_{\Lambda_{n}}\right)+\left|A_{n}\right|^{-1} Q_{n}^{F}\left(F_{n}\right) .
$$

We thus seek a lower bound of the expressions on the right. To deal with the first term we introduce the measure

$$
\widetilde{Q}_{n}^{F}=\left|A_{n}\right|^{-1} \int_{A_{n}} \widehat{Q}_{n}^{F} \circ \vartheta_{x}^{-1} d x
$$

where $\hat{Q}_{n}^{F}=\underset{i \in p_{n} \mathbb{Z}^{d}}{*} Q_{n}^{F} \circ \vartheta_{i}^{-1}$ is the shift-periodic point random field with period $p_{n}=\left|A_{n}\right|^{1 / d}$ relative to which the configurations in the disjoint blocks $A_{n}+i$, $i \in p_{n} \mathbb{Z}^{d}$, are iid with distribution $Q_{n}^{F}$.

Lemma 5.5 For all $n, \widetilde{Q}_{n}^{F} \in \mathscr{P}_{\mathbb{B}}$ and $I\left(\widetilde{Q}_{n}^{F}\right) \leqq\left|\Lambda_{n}\right|^{-1} I\left(Q_{n}^{F} ; Q_{\Lambda_{n}}\right)$.

As a matter of fact, the preceding inequality is even an identity, as can be shown using convex analysis or an analogue of Theorem 15.20 of [7]. We do not need this fact here. 
Proof. Clearly, $\widetilde{Q}_{n}^{F}$ is stationary. Moreover, since $F_{n} \geqq 0$ we have $Q_{n}^{F} \leqq c_{n} Q_{A_{n}}$ for some $c_{n}<\infty$ and thus

$$
\widetilde{Q}_{n}^{F}\left(N_{A_{n}}^{\psi}\right)=Q_{n}^{F}\left(N_{A_{n}}^{\psi}\right) \leqq c_{n} Q\left(N_{A_{n}}^{\psi}\right)=c_{n}\left|A_{n}\right| \mu(\psi)<\infty .
$$

Hence $\widetilde{Q}_{n}^{F}$ has finite $\psi$-intensity.

In view of the product structure of $\hat{Q}_{n}^{F} \circ \vartheta_{x}^{-1}, I\left(\hat{Q}_{n}^{F} \circ \vartheta_{x}^{-1}\right)$ (as defined by the limit (2.12)) exists and equals $a_{n}=\left|\Lambda_{n}\right|^{-1} I\left(Q_{n}^{F} ; Q_{\Lambda_{n}}\right)$; cf. the proof of Proposition 16.34 of [7]. It is also easily seen that for each $\Delta \in \mathscr{C}$ and $x \in S$

$$
I\left(\left(\hat{Q}_{n}^{F} \circ \vartheta_{x}^{-1}\right)_{\Delta} ; Q_{A}\right) \leqq\left|\Delta^{+, n}\right| a_{n},
$$

where $A^{+, n}=\left\{y \in S: d_{S}(y, \Delta) \leqq \operatorname{diam} A_{n}\right\}$. Letting $\Delta$ run through the sequence $\left(A_{k}\right)$ we thus conclude from the dominated convergence theorem that

$$
a_{n}=\lim _{k \rightarrow \infty}\left|\Lambda_{k}\right|^{-1}\left|\Lambda_{n}\right|^{-1} \int_{A_{n}} I\left(\left(\hat{Q}_{n}^{F} \circ \vartheta_{x}^{-1}\right)_{A_{k}} ; Q_{A_{k}}\right) d x .
$$

By (2.11), relative entropy is a measure convex function of its first argument. The last expression is therefore not less than

$$
\lim _{k \rightarrow \infty}\left|A_{k}\right|^{-1} I\left(\left(\widetilde{Q}_{n}^{F}\right)_{\Lambda_{k}} ; Q_{\Lambda_{k}}\right)=I\left(\widetilde{Q}_{n}^{F}\right) .
$$

This proves the second assertion of the lemma.

To estimate the second term on the right side of (5.5) we introduce the lower convex envelope

$$
\underline{F}=\sup \left\{e_{f}: f \in \mathscr{L}, e_{f}<F\right\}
$$

of $F$.

Lemma 5.6 For all $n$ we have

$$
\left|\Lambda_{n}\right|^{-1} Q_{n}^{F}\left(F_{n}\right) \geqq \underline{F}\left(Q_{n}^{F} R_{n}\right)-\delta_{n}^{F}\left(1+q_{n}^{F}\right),
$$

where $Q_{n}^{F} R_{n}=\int R_{n, \omega} Q_{n}^{F}(d \omega) \in \mathscr{P}_{\boldsymbol{\theta}}$ and $q_{n}^{F}=z^{\psi}\left(Q_{n}^{F} R_{n}\right)$.

Proof. Since all $R_{n, \omega}$ are stationary, so is their mixture $Q_{n}^{F} R_{n}$. Its $\psi$-intensity is given by

$$
z^{\psi}\left(Q_{n}^{F} R_{n}\right)=Q_{n}^{F}\left(z^{\psi}\left(R_{n}\right)\right)=\left|\Lambda_{n}\right|^{-1} Q_{n}^{F}\left(N_{\Lambda_{n}}^{\psi}\right)
$$

which is finite because $F_{n} \geqq 0$. Thus $Q_{n}^{F} R_{n} \in \mathscr{P}_{\theta}$. Also, for each $f \in \mathscr{L}$ with $e_{f}<F$ we obtain from (3.1) that

$$
\left|\Lambda_{n}\right|^{-1} F_{n} \geqq R_{n}(f)-\delta_{n}^{F}\left(1+z^{\psi}\left(R_{n}\right)\right) .
$$

Integrating this with respect to $Q_{n}^{F}$ and taking the supremum over $f$ we arrive at the stated inequality.

The next step consists in showing that the measures $\widetilde{Q}_{n}^{F}$ and $Q_{n}^{F} R_{n}$ are asymptotically identical. 
Lemma 5.7 Suppose that $\lim \sup I\left(\widetilde{Q}_{n}^{F}\right)<\infty$. Then

$$
\begin{aligned}
& \lim _{n \rightarrow \infty}\left[\widetilde{Q}_{n}^{F}(f)-Q_{n}^{F} R_{n}(f)\right]=0
\end{aligned}
$$

for all $f \in \mathscr{L}$.

Proof. Let $f \in \mathscr{L}$ be given and $\Delta \in \mathscr{C}$ be such that $f$ is $r_{A}^{-1} \mathscr{F}_{A}$-measurable and $|f| \leqq b\left(1+N_{\Delta}^{\psi}\right)$ for some $b<\infty$. Proceeding as in the proof of Remark 2.4 we then find that

$$
\begin{aligned}
& \left|\widetilde{Q}_{n}^{F}(f)-Q_{n}^{F} R_{n}(f)\right| \\
& \quad \leqq\left|\Lambda_{n}\right|^{-1} \int_{\partial \Lambda_{n}}\left[\hat{Q}_{n}^{F}\left(\left|f \circ \vartheta_{x}\right|\right)+\int\left|f\left(\vartheta_{x} \omega^{(n)}\right)\right| Q_{n}^{F}(d \omega)\right] d x \\
& \quad \leqq 2 b\left|\Lambda_{n}\right|^{-1}\left|\partial \Lambda_{n}\right|+2 b\left|\Lambda_{n}\right|^{-1} \int_{\partial \Lambda_{n}} \hat{Q}_{n}^{F}\left(N_{\Delta+x}^{\psi}\right) d x
\end{aligned}
$$

because

$$
\int N_{\Delta+x}^{\psi}\left(\omega^{(n)}\right) Q_{n}^{F}(d \omega)=Q_{n}^{F}\left(N_{\Delta_{x, n}}^{\psi}\right)=\hat{Q}_{n}^{F}\left(N_{\Delta+x}^{\psi}\right) .
$$

For any $a>0$ we may write further

$$
\begin{aligned}
& \left|A_{n}\right|^{-1} \int_{\partial A_{n}} \hat{Q}_{n}^{F}\left(N_{\Delta+x}^{\psi}\right) d x \\
& \quad \leqq a\left|\Lambda_{n}\right|^{-1}\left|\partial A_{n}\right|+\left|\Lambda_{n}\right|^{-1} \int_{A_{n}} \hat{Q}_{n}^{F}\left(N_{\Delta+x}^{\psi} 1_{\left\{N_{\Delta+x}^{\psi}>a\right\}}\right) d x \\
& \quad=a\left|A_{n}\right|^{-1}\left|\partial A_{n}\right|+\widetilde{Q}_{n}^{F}\left(N_{\Delta}^{\psi} 1_{\left\{N_{\Delta}^{\psi}>a\right\}}\right) .
\end{aligned}
$$

By Lemma 5.2, the last term can be made arbitrarily small (uniformly in $n$ ) by choosing a sufficiently large. The lemma thus follows by letting first $n \rightarrow \infty$ and then $a \rightarrow \infty$.

\section{Lemma 5.8}

$$
\limsup _{n \rightarrow \infty}\left|\Lambda_{n}\right|^{-1} \log Q_{\Lambda_{n}}\left(e^{-F_{n}}\right) \leqq-\inf [I+F]
$$

Proof. Suppose the contrary. Then there exists a number $c<\inf [I+\underline{F}]$ such that

$$
-\left|\Lambda_{n}\right|^{-1} \log Q_{\Lambda_{n}}\left(e^{-F_{n}}\right)<c
$$

for infinitely many $n$. Equation (5.5) and Lemmas 5.5 and 5.6 then imply that

$$
I\left(\widetilde{Q}_{n}^{F}\right)+\underline{F}\left(Q_{n}^{F} R_{n}\right)-\delta_{n}^{F}\left(1+q_{n}^{F}\right)<c
$$

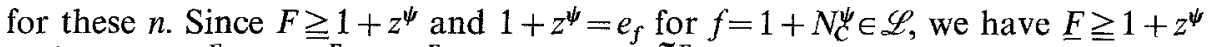
and thus $\underline{F}\left(Q_{n}^{F} R_{n}\right)-\delta_{n}^{F}\left(1+q_{n}^{F}\right) \geqq 0$. Hence $I\left(\widetilde{Q}_{n}^{F}\right)<c$ for infinitely many $n$. Proposition 2.6 now implies the existence of a subsequence $\left(\widetilde{Q}_{n_{k}}^{F}\right)_{k \geqq 1}$ which converges to some $P \in\{I \leqq c\}$. By Lemma 5.7, $P$ is also the limit of the sequence $\left(Q_{n_{k}}^{F} R_{n_{k}}\right)_{k \geqq 1}$. Hence $q_{n_{k}}^{F} \rightarrow z^{\psi}(P)<\infty$ and therefore $\delta_{n_{k}}^{F}\left(1+q_{n_{k}}^{F}\right) \rightarrow 0$ as $k \rightarrow \infty$. Letting $n=n_{k} \rightarrow \infty$ 
in (5.8) and using the lower semicontinuity of $I$ and $\underline{F}$ we thus find that $I(P)$ $+\underline{F}(P) \leqq c$, in contradiction to the choice of $c$.

The upper bound (3.2) follows immediately from the last and the next lemma.

Lemma $5.9 \inf [I+\underline{F}]=\inf \left[I+F_{1 \mathrm{sc}}\right]$.

The proof of the corresponding Lemma 4.5 in [8] only rests on some general structural properties which hold also in the present setting.

We now turn to the lower bound (3.3). Its proof follows a standard device which is outlined in [5], for example. We mention some details because additional care is needed. We must show that

$$
\liminf _{n \rightarrow \infty}\left|\Lambda_{n}\right|^{-1} \log Q_{\Lambda_{n}}\left(e^{-F_{n}}\right) \geqq-\left[I(P)+F^{\mathrm{lsc}}(P)\right]
$$

for each $P \in \mathscr{P}_{\Theta}$ for which the expression on the right is finite. We can assume that $P$ is ergodic relative to $\Theta$. Indeed, if $P_{n}$ is defined by formula (5.6) with $Q_{n}^{F}$ replaced by $P_{A_{n}}$ then $I\left(P_{n}\right) \leqq I(P)$ by an analogue of Lemma 5.5, and thus $P_{n} \rightarrow P$ by an analogue of Lemma 5.7 combined with Remark 2.5 . Thus for each $\varepsilon>0$ there is some $n$ with

$$
I(P)+F^{\mathrm{lsc}}(P)+\varepsilon>I\left(P_{n}\right)+F^{\mathrm{usc}}\left(P_{n}\right),
$$

and it is easily seen that each $P_{n}$ is ergodic.

Next, it follows from (2.8) and McMillan's theorem in the version of Fritz [6] or Nguyen and Zessin [13] that, for each $\varepsilon>0, P_{A_{n}}\left(A_{n, \varepsilon}\right) \rightarrow 1$ as $n \rightarrow \infty$, where

$$
A_{n, \varepsilon}=\left\{F\left(R_{n}\right)<F^{\mathrm{usc}}(P)+\varepsilon, d P_{A_{n}} / d Q_{\Lambda_{n}} \leqq \exp \left|\Lambda_{n}\right|(I(P)+\varepsilon)\right\} .
$$

Thus we can write, using Jensen's inequality in the last step,

$$
\begin{aligned}
& \left|A_{n}\right|^{-1} \log Q_{\Lambda_{n}}\left(e^{-F_{n}}\right) \geqq\left|A_{n}\right|^{-1} \log Q_{A_{n}}\left(e^{-F_{n}} 1_{A_{n_{n}}}\right) \\
& \quad \geqq-F^{\mathrm{usc}}(P)-I(P)-2 \varepsilon+\left|A_{n}\right|^{-1} \log P_{A_{n}}\left(e^{-\delta_{n}^{F}\left|A_{n}\right|\left(1+z^{\psi}\left(R_{n}\right)\right)} 1_{A_{n, \varepsilon},}\right) \\
& \quad \geqq-F^{\mathrm{usc}}(P)-I(P)-2 \varepsilon-\delta_{n}^{F}\left(1+z^{\psi}(P) P_{\Lambda_{n}}\left(A_{n, \varepsilon}\right)^{-1}\right)+\left|A_{n}\right|^{-1} \log P_{A_{n}}\left(A_{n, \varepsilon}\right) .
\end{aligned}
$$

In the limit $n \rightarrow \infty, \varepsilon \rightarrow 0$ we arrive at (5.9).

\subsection{The maximum entropy principle}

In this final subsection we shall prove Theorem 3.3. As we have stated in the introduction, this proof is based on the very same ideas as that of the upper bound (3.2). First of all, it follows from (3.3) and hypothesis (3.6) that $Q_{A_{n}}\left(e^{-F_{n}}\right)$ $>0$ eventually. The measures $Q_{n}^{F}$ in (3.5) are thus well-defined for all sufficiently large $n$. Next, we can again assume without loss that $F \geqq 1+z^{\psi}$ and $F_{n} \geqq 0$ for these $n$. For, if $\left\{\left(F_{n}^{*}\right), F^{*}\right\}$ and $Q^{*}$ are defined as in Lemma 5.4 then $\left(Q^{*}\right)_{n}^{F^{*}}=Q_{n}^{F}$ for all $n$ for which $Q_{n}^{F}$ is defined, and $\left(M^{*}\right)^{F^{*}}=M^{F}$ because $I^{*}+F_{1 \mathrm{sc}}^{*}$ and $I+F_{\mathrm{lsc}}$ differ only by a finite constant; cf. the proof of Lemma 5.4.

By Eq. (5.5) and Lemmas 5.5 and 5.6,

$$
I\left(\widetilde{Q}_{n}^{F}\right)+\underline{F}\left(Q_{n}^{F} R_{n}\right)-\delta_{n}^{F}\left(1+q_{n}^{F}\right) \leqq-\left|A_{n}\right|^{-1} \log Q_{\Lambda_{n}}\left(e^{-F_{n}}\right) .
$$


Combining this inequality with the lower bound (3.3), hypothesis (3.6), and Lemma 5.9 we obtain

$$
\begin{aligned}
& \limsup _{n \rightarrow \infty}\left[I\left(\widetilde{Q}_{n}^{F}\right)+\underline{F}\left(Q_{n}^{F} R_{n}\right)-\delta_{n}^{F}\left(1+q_{n}^{F}\right)\right] \\
& \quad \leqq \inf [I+\underline{F}]<\infty .
\end{aligned}
$$

Since $F \geqq 1+z^{\psi}$ and thus $\underline{F} \geqq 1+z^{\psi}$ and $\delta_{n}^{F} \leqq 1$ eventually, this implies that $\tilde{Q}_{n}^{F}$ eventually belongs to a level set of $I$. Proposition 2.6 thus shows that a suitable subsequence $\widetilde{Q}_{n_{k}}^{F}$ converges to some $Q_{\infty}^{F} \in \mathscr{P}_{\theta}$. For any such subsequence $\left(n_{k}\right)$ and $Q_{\infty}^{F}$ we conclude from Lemma 5.7 that also $Q_{n_{k}}^{F} R_{n_{k}} \rightarrow Q_{\infty}^{F}$, and thereby $q_{n_{k}}^{F}$ $\rightarrow z^{\psi}\left(Q_{\infty}^{F}\right)<\infty$, as $k \rightarrow \infty$. Since $I$ and $\underline{F}$ are lower semicontinuous, we therefore obtain from (5.10) that $I\left(Q_{\infty}^{F}\right)+\underline{F}\left(Q_{\infty}^{F}\right) \leqq \inf [I+\underline{F}]$. That is, $Q_{\infty}^{F}$ belongs to the compact set $\{I+E=\min \}$. By Lemma 4.8 of [8] this set consists of all barycenters of Borel probability measures on $M^{F}=\left\{I+F_{\mathrm{lsc}}=\min \right\}$. Therefore it only remains to observe that the sequences $\left(\widetilde{Q}_{n}^{F}\right)$ and $\left(Q_{n}^{F}\right)$ have the same set of accumulation points. This follows from Lemma 5.7 and the fact that, under our hypotheses on $F_{n}, Q_{n}^{F} R_{n}=Q_{n}^{F}$ on $r_{A_{n}}^{-1} \mathscr{F}_{A_{n}}$; cf. Lemma 4.6 of [8]. The proof of Theorem 3.3 is thus complete.

\section{References}

1. Csiszár, I.: Sanov property, generalized $I$-projection and a conditional limit theorem. Ann. Probab. 12, 768-793 (1984)

2. Daley, D.J., Vere-Jones, D.: An introduction to the theory of point processes. Berlin Heidelberg New York: Springer 1988

3. Deuschel, J.D., Stroock, D.W., Zessin, H.: Microcanonical distributions for lattice gases. Commun. Math. Phys. 139, 83-101 (1991)

4. Ellis, R.S., Newman, C.M.: Necessary and sufficient conditions for the GHS inequality with applications to probability and analysis. Trans. Am. Math. Soc. 237, 83-99 (1978)

5. Föllmer, H.: Random fields and diffusion processes. In: Hennequin, P.L. (ed.) Ecole d'Eté de Probabilités de Saint-Flour XV-XVII. (Lect. Notes Math., vol. 1362, pp. 101-203) Berlin Heidelberg New York: Springer 1988

6. Fritz, J.: Generalization of McMillan's theorem to random set functions. Stud. Sci. Math. Hung. 5, 369-394 (1970)

7. Georgii, H.O.: Gibbs measures and phase transitions. Berlin: de Gruyter 1988

8. Georgii, H.O.: Large deviations and maximum entropy principle for interacting random fields on $Z^{d}$. Ann. Probab. (to appear, 1993)

9. Georgii, H.O.: Large deviations for hard-core particle systems. In: Kotecky, R. (ed.) Proceedings of the 1992 Prague Workshop on Phase Transitions. Singapore: World Scientific 1993

10. Krickeberg, K.: Processes ponctuels en statistique. In: Hennequin, P.L. (ed.) Ecole d'Eté de Probabilités de Saint-Flour X. (Lect. Notes Math., vol. 929, pp. 206-313) Berlin Heidelberg New York: Springer 1980

11. Matthes, K., Kerstan, J., Mecke, J.: Infinitely divisible point processes. Chichester: Wiley 1978

12. Mecke, J.: Stationäre zufällige Maße auf lokalkompakten abelschen Gruppen. Z. Wahrscheinlichkeitstheor. Verw. Geb. 9, 36-58 (1967)

13. Nguyen, X.X., Zessin, H.: Ergodic theorems for spatial processes. Z. Wahrscheinlichkeitstheor. Verw. Geb. 48, 133-158 (1979)

14. Olla, S., Varadhan, S.R.S., Yau, H.T.: Hydrodynamic limit for a Hamiltonian system with a weak noise (Preprint 1992)

15. Papangelou, F.: On the entropy rate of stationary point processes and its discrete approximation. Z. Wahrscheinlichkeitstheor. Verw. Geb. 44, 191-211 (1978) 
16. Rockafellar, R.T.: Convex analysis. Princeton: Princeton University Press 1970

17. Roelly, S., Zessin, H.: The equivalence of equilibrium principles in statistical mechanies and some applications to large particle systems. Expos. Math. (to appear, 1993)

18. Ruelle, D.: Existence of a phase transition in a continuous classical system. Phys. Rev. Lett. 27, 1040-1041 (1971)

19. Varadhan, S.R.S.: Large deviations and applications. In: Hennequin, P.L. (ed.) Ecole d'Eté de Probabilités de Saint Flour XV-XVII. (Lect. Notes Math., vol. 1362, pp. 1-49) Berlin Heidelberg New York: Springer 1988

20. Widom, B., Rowlinson, J.S.: New model for the study of liquid-vapor phase transitions. J. Chem. Phys. 52, 1670-1684 (1970)

21. Zessin, H.: Boltzmann's principle for Brownian motion on the torus. In: Blanchard, Ph. (ed.) Dynamics of complex and irregular systems. Singapore: World Scientific 1993 